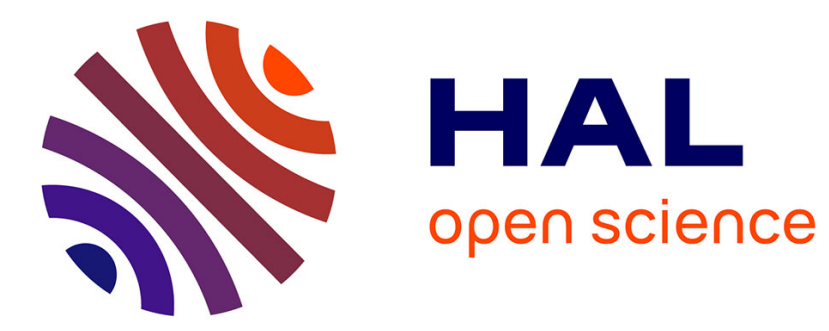

\title{
Evolutionary strategic beliefs and financial markets
}

Elyès Jouini, Clotilde Napp, Yannick Viossat

\section{To cite this version:}

Elyès Jouini, Clotilde Napp, Yannick Viossat. Evolutionary strategic beliefs and financial markets.

Review of Finance, 2012, pp.1. halshs-00556490

\section{HAL Id: halshs-00556490 \\ https://shs.hal.science/halshs-00556490}

Submitted on 17 Jan 2011

HAL is a multi-disciplinary open access archive for the deposit and dissemination of scientific research documents, whether they are published or not. The documents may come from teaching and research institutions in France or abroad, or from public or private research centers.
L'archive ouverte pluridisciplinaire HAL, est destinée au dépôt et à la diffusion de documents scientifiques de niveau recherche, publiés ou non, émanant des établissements d'enseignement et de recherche français ou étrangers, des laboratoires publics ou privés. 


\title{
Evolutionary Strategic Beliefs and Financial Markets*
}

\author{
Elyès Jouini \\ CEREMADE, Université Paris Dauphine, \\ Place du Maréchal de Lattre de Tassigny, \\ 75775 Paris cedex 16, \\ jouini@ceremade.dauphine.fr; \\ phone: + 331440542 26; fax: + 33144054849 . \\ Clotilde Napp \\ CNRS \& Université Paris Dauphine, \\ Place du Maréchal de Lattre de Tassigny, \\ 75775 Paris cedex 16; \\ clotilde.napp@dauphine.fr, \\ phone: + 331440546 42; fax: + 33144054849 \\ Yannick Viossat \\ CEREMADE, Université Paris Dauphine, \\ Place du Maréchal de Lattre de Tassigny, \\ 75775 Paris cedex 16; \\ viossat@ceremade.dauphine.fr, \\ phone: + 331440546 71; fax: + 33144054599
}

December 8, 2010

\begin{abstract}
We provide a discipline for belief formation through a model of subjective beliefs, in which agents hold strategic beliefs. More precisely, we consider beliefs as a

*The financial support of the GIP-ANR and of the Risk Foundation (Groupama Chair) is gratefully acknowledged by the authors. We have benefitted from comments from participants at the Parisian Seminar of Game Theory (Paris), Bachelier Seminar (Paris) and at the Adam Smith Asset Pricing Conference (LBS) and in particular Françoise Forges, Denis Gromb, Marco Ottaviani, Sylvain Sorin,
\end{abstract} Dimitri Vayanos. 
strategic variable that agents can choose (consciously or not) in order to maximize their utility at the equilibrium. These strategic beliefs result from an evolutionary process. We find that evolutionary strategic behavior leads to belief subjectivity and heterogeneity. Optimism (resp. overconfidence) as well as pessimism (resp. doubt) both emerge from the evolution process. Furthermore, we obtain a positive correlation between pessimism (resp. doubt) and risk-tolerance. We analyze the equilibrium characteristics. Under reasonable assumptions, the consensus belief is pessimistic and, as a consequence, the risk premium is higher than in a standard setting.

Keywords: beliefs formation, strategic beliefs, optimal beliefs, distorded beliefs, pessimism, risk premium

JEL codes: G12, D03, D53, D81 


\title{
Evolutionary Strategic Beliefs and Financial Markets
}

\begin{abstract}
We provide a discipline for belief formation through a model of subjective beliefs, in which agents hold strategic beliefs. More precisely, we consider beliefs as a strategic variable that agents can choose (consciously or not) in order to maximize their utility at the equilibrium. These strategic beliefs result from an evolutionary process. We find that evolutionary strategic behavior leads to belief subjectivity and heterogeneity. Optimism (resp. overconfidence) as well as pessimism (resp. doubt) both emerge from the evolution process. Furthermore, we obtain a positive correlation between pessimism (resp. doubt) and risk-tolerance. We analyze the equilibrium characteristics. Under reasonable assumptions, the consensus belief is pessimistic and, as a consequence, the risk premium is higher than in a standard setting.
\end{abstract}

Keywords: beliefs formation, strategic beliefs, optimal beliefs, distorded beliefs, pessimism, risk premium

JEL codes: G12, D03, D53, D81 


\section{Introduction}

In the classical financial economics theory, decision makers are assumed to have homogenous and rational expectations. This assumption has been the basis for many developments in finance. Among these developments, the portfolio selection model (Markowitz, 1952) and the Capital Asset Pricing Model (CAPM, Sharpe 1964 and Lintner 1965) play an important role. Given their simplicity and empirical tractability, these models and their subsequent extensions have become a significant cornerstone of theoretical and applied economics from insurance and financial theory to the theory of the firm.

However, the last 30 years have seen an accumulation of empirical tests that invalidate the theoretical conclusions of these models based on the assumption of homogeneous and objective beliefs. The homogeneous prior beliefs assumption (Harsanyi doctrine) is weaker than the assumption of rational expectations that all agents' prior beliefs are equal to the objective probabilities. But like rational expectations, the common priors assumption is quite restrictive and does not allow agents to "agree to disagree" (Aumann, 1976). It suffices to observe the heterogeneity of analysts or professional forecasters forecasts or more generally of experts opinions to realize that this assumption is not realistic. Savage (1954) provides axiomatic foundations for a more general theory in which agents hold arbitrary prior beliefs, so agents can agree to disagree. But the alternative to rational expectations lacks discipline and if beliefs can be arbitrary, theory provides little structure or predictive power. Indeed, once the assumption of rational and homogenous expectations is relaxed, the following questions arise : How do agents form their beliefs? Do beliefs exhibit optimism? pessimism? overconfidence? doubt (or underconfidence)? How are these possible biases related to the agents preferences? How are agents' beliefs affected by strategic interaction? What is the impact of these beliefs or expectations on individual decisions and on equilibrium characteristics such as prices, risk premium and risk-sharing?

Our aim is to provide a rationale for belief heterogeneity that enlightens the reflexion about the questions above. In our model, the way beliefs are formed is dynamic and obeys evolutionary rules. Asymptotically, this leads to a situation in which agents hold incorrect but strategic beliefs in the sense that each agent acts as if her beliefs were a strategic variable that she can choose (consciously or not) to maximize her utility from trade. Such strategic beliefs emerge as the result of the selection pressure towards agents that are born with such beliefs, or by agents gradually learning that a certain way of forming beliefs is more rewarding than other ways. This is why we call them evolutionary strategic beliefs.

Sandroni (2000) gives a justification for rational expectations equilibria, showing that a market populated by agents who initially differ in the accuracy of their predictions will nonetheless converge to a competitive rational expectations equilibrium as those agents who make inaccurate predictions are driven out of the market by those who are more 
accurate. This argument is in the same vein as the arguments of Alchian (1950) and Friedman (1953) applied to the profit maximization assumption in a competitive market. In contrast, we show through evolutionary arguments that strategic interaction leads to heterogeneous and subjective beliefs. More precisely, we analyze the situation where the agents learn from interaction in the sense that they dynamically modify the frequencies at which they play the different possible strategies, underweighting the strategies that lead to low utility levels and overweighting the strategies leading to high utility levels. In fact, each agent is represented as a population of individuals each programmed to play a given strategy. We use then concepts from evolutionary game theory where strategies with high payoff spread within the population, the payoffs depending on the frequencies of the strategies within the population. We show that the asymptotical behavior of our agents corresponds to the behaviour that they would adopt in a static game that is naturally associated to our evolutionary process. This game corresponds to a situation where each agent adopts a belief to maximize his utility from trade, taking into account the effect his choice has on price and taking as given the strategy of the other agents. The asymptotic behaviour of our agents corresponds then to a Nash equilibrium in demands, as presented in Kyle ${ }^{1}$ (1989). An agent who forms beliefs strategically forms beliefs that are (the most) useful to him. She takes what pays rather than what is true. The idea that in strategic situations players may gain from having an objective function different from actual payoff maximization dates back to Schelling (1960). It has been more recently developed in a fairly general framework by Heifetz et al. (2007a,b). The main contribution of our paper is to apply this idea to beliefs formation in a financial markets framework. We want to characterize the types of beliefs that survive especially in terms of optimism/overconfidence and to analyze the implications in terms of equilibrium characteristics.

Our strategic explanation of beliefs should be contrasted with 'rational' approaches to beliefs where agents try to reflect the 'world as it is' in their beliefs. We also contrast the strategic explanation of beliefs to approaches in which forward-looking agents optimally distort beliefs and in which beliefs are of intrinsic value to agents, as with wishful thinking, self-esteem or fear of disappointment (Akerlof-Dickens, 1982, Benabou-Tirole, 2002, Brunnermeier-Parker, 2005, Gollier-Muermann, 2010). In these models, beliefs result from an individual optimization problem while our model of belief formation is strategic.

Our evolutionary strategic beliefs concept is similar to the pragmatic beliefs concept

\footnotetext{
${ }^{1}$ As underlined by Kyle "(this) is perhaps the most obvious modification of the conventional competitive rational expectations concept. It preserves market clearing through a Walrasian mechanism and keeps the Nash flavour of a competitive equilibrium." In fact, the concept of Nash equilibrium in demand schedules is the analogon, from the consumers point of view, of the Cournot-Nash equilibrium in supply for producers.
} 
of Hvide (2002) in the sense that in both cases, agents select the beliefs that are more beneficial for them. In fact, the notion of pragmatic beliefs refers to the philosophical school known as Pragmatism. Russell (1945) interprets one of its main ideas as follows: agents should (or do) hold beliefs that have good consequences. Note that Hvide (2002) uses this concept in a framework that is very different from ours. More precisely, he considers a principal-agent model in a job market framework where agents have (pragmatically built) beliefs about their ability.

Our model is embedded in a simple, standard equilibrium problem with risky assets and beliefs are about the risk distribution. Its structure may be applied to several problems in which risk-averse agents have to choose the optimal exposure to a risk. This is the case, for example, when individuals interact on a financial market or when an insurance company has to negociate an optimal retention rate with a reinsurance company or when entrepreneurs have to fix the optimal proportion of equity to retain for a given project.

Our findings are the following. Our evolutionary approach leads to belief subjectivity and heterogeneity. This means that in a standard portfolio/equilibrium problem in which beliefs are determined through an evolution process, the objective belief is not optimal, and agents differ in their beliefs. Indeed, optimism (resp. overconfidence) as well as pessimism (resp. doubt) both survive in the long run. Furthermore, we find a positive correlation between pessimism (resp. doubt) and risk-tolerance. The intuition is as follows. For a relatively risk-tolerant agent, his demand in the risky asset is positive, so that his expected utility from trade is decreasing in the price of the risky asset. A pessimistic belief is associated to a lower demand, hence to a lower price, and balances this benefit of pessimism against the costs of worse decision making. The converse reasoning applies to a very risk-averse agent, who, at the equilibrium, has a negative demand in the risky asset and benefits from optimism. Such a positive correlation has been observed in empirical studies in a purely behavioural setting (Ben Mansour et al., 2008) ${ }^{2}$.

In an exponential utility and normal distribution setting, the consensus belief (or the representative agent belief), which is given by the average of the individual beliefs weighted by the risk-tolerance, is pessimistic (resp. exhibits doubt). Intuitively, the more risk-tolerant agents make the market, and the consensus belief reflects the characteristics of the more risk-tolerant. Since we have just seen that the more risk-tolerant agents are pessimistic, it is consistent to obtain a pessimistic consensus belief. Moreover, the average (unweighted) belief is also pessimistic, which means that the pessimistic risktolerant agents are more pessimistic than the optimistic are optimistic and there is then a pessimistic bias in individual beliefs. Such a pessimistic bias has been observed in

\footnotetext{
${ }^{2}$ This might seem counterintuitive because the two concepts (pessimism and risk-aversion) appear at first sight as closely related. Investing a large amount in a risky asset may result indifferently from optimism or risk-tolerance. Similarly an entrepreneurial behavior may be explained by either concepts. However, this does not provide any hint about the complementarity or the substituability of these two concepts.
} 
empirical studies in a purely behavioural setting (Ben Mansour et al., 2006), in a decision theory framework (Wakker, 2001) or in a market framework (Giordani and Söderlind, 2006). In particular, as underlined by Shefrin (2005) based on Wall \$treet Week data "between 1983 and 2002, professional investors were unduly pessimistic, underestimating market returns".

One may argue that various empirical studies of professionals' economic forecasts as well as psychological surveys have opposite conclusions: optimism (see e.g. Fried and Givoly, 1982, O’Brien, 1988, Francis and Philbrick, 1993, Kang et al., 1994 and Dreman and Berry, 1995) and overconfidence (Rabin, 1998, Hirshleifer, 2001, Giordani and Söderlind, 2006). However, it has been repeatedly argued in the literature that professionals' forecasts may be biased by environmental factors (see e.g. Schipper, 1991, Mc Nichols and O'Brien, 1997, Darrough and Russell, 2002). Furthermore, these studies are generally based on self-assessment. For instance, in psychology, personal pessimism measures how individuals perceive their future while it is clear that individuals have an influence on this future. When this self assessment dimension disappears, it has been shown in various studies that the optimistic bias disappears too ${ }^{3}$. Finally, Ben Mansour et al. (2006) have shown that the optimistic bias is transformed into a pessimistic bias when we focus on how individuals perceive the future through items that do have a clear direct impact on their well-being but on which they have no influence. This is coherent with the fact that people tend to attribute success to their own actions but failure to external factors (Zuckerman, 1979, Fiske and Taylor, 1991, Baumeister, 1998, Duval and Silvia, 2002, Van den Steen, 2004).

It is interesting from this perspective to note that pragmatic beliefs also lead to overconfidence in Hvide (2002) (while they lead to underconfidence in our framework). Hvide (2002) focuses on overconfidence in the sense of "hubris" since agents have beliefs about their own ability while we focus on overconfidence in the sense of excessive confidence in the statistical sense (as opposed to doubt) since agents have beliefs about external sources of risk ${ }^{4}$. The difference between these two forms of overconfidence is of the same nature as the difference between the two forms of pessimism (with or without self-assessment).

Our results obtained in a strategic interaction framework differ from those obtained in an optimal beliefs/illusions setting, in which in most cases there is no belief heterogeneity and an optimistic bias ${ }^{5}$ (Brunnermeier-Parker, 2005, Brunnermeier et al., 2007, Gollier,

\footnotetext{
${ }^{3}$ For instance, Wenglert and Rosen (2000) conclude to optimism for items like I will have a happy life, I will keep my best friends and to neutrality for items like There will be a third world war, The unemployment rate shall fall, Life expectancy shall increase.

${ }^{4}$ Hvide (2002) makes such a distinction and refers to these two possible definitions for overconfidence as overconfidence $_{1}$ and overconfidence ${ }_{2}$ and then adopts overconfidence . $_{5}$

${ }^{5}$ Gollier-Muermann (2010) consider a model of optimal beliefs with ex ante savoring and ex post disappointment. Depending upon the intensity of anticipatory feelings and disappointment they might
} 
2005). This bias results from the specific mental process they consider. Indeed, in these models, subjective beliefs maximize the agents' expected well-being defined as the time average of expected felicity over all periods. Since agents that care about future utility flows have a higher current felicity if they are optimistic, the optimal beliefs balance this benefit of optimism against the costs of worse decision making.

As a consequence of the pessimistic bias at the aggregate level, our evolutionary approach leads to a the risk premium that is greater than in the standard rational expectations equilibrium. The fact that a pessimistic bias and a positive correlation between risk tolerance and pessimism lead to an increase of the risk premium has been underlined by Abel (1989), Calvet et al. (2002), Detemple-Murthy (1994), Gollier (2007) and JouiniNapp (2006); in their models, beliefs are exogenously given while one of the main features of this paper is to construct a model in which beliefs are endogeneous and in which the pessimistic bias and the positive correlation between risk tolerance and pessimism, both emerge at the equilibrium.

This increase of the risk premium is interesting in light of the risk premium puzzle (Mehra and Prescott, 1985). In the insurance industry, our results lead to a situation where the more risk-averse agent (the insured) is optimistic and the less risk-averse agent (the insurer) is pessimistic. The average belief is pessimistic leading to a higher insurance premium, which might help to explain the purchase of vastly overpriced insurance in a range of situations (Cutler and Zeckhauser, 2004). In corporate finance, IPOs can be modeled as a decision for a risk-averse entrepreneur to sell shares of his firm to more risktolerant investors. The application of our results to such a setting leads to a pessimistic consensus belief. As a result, the firm is underpriced and the short run return is large, which is consistent with the empirical literature on IPOs (Ibbotson and Ritter, 1995). Obviously, we don't pretend that strategic interaction, such as in our simple model, is the unique explanation for these puzzles, however, it is interesting to remark that our approach helps to explain these puzzles as well as belief heterogeneity without introducing any information asymmetry nor principal-agent features.

The paper is organized as follows. Section 2 introduces the concept of evolutionary strategic beliefs and details the dynamics of beliefs formation. We also explain the link between the evolutionary framework and a static concept of Nash equilibrium in demand schedules. In Section 3, explicit computations are provided in a setting with exponential utility functions and normal distributions in which the strategic variable is the expected payoff of the risky asset. In Section 4, we compare our results with those obtained in an optimal beliefs setting. In Section 5, qualitative results are provided in a setting with more general utility functions and distributions. Section 6 considers extensions of the model of Section 3 in essentially two directions; a model in which the strategic variable 
is the variance of the payoff of the risky asset and a model with multiple sources of risk. Section 7 concludes.

\section{A model of evolutionary strategic beliefs}

We consider a standard equilibrium model, except that we allow for a possible evolution of individual beliefs through strategic interaction. We start by describing the Walrasian equilibrium. We then introduce the evolutionary framework as well as the underlying 2-player game.

\subsection{The Walrasian equilibrium}

The economy is composed of two agents with real valued, increasing and strictly concave utility functions $u_{1}$ and $u_{2}$ defined on $\mathbb{R}_{+}$. The agents live for one period and consumption takes place at the end of the period. The states of nature are described by a probability space $(\Omega, F, P)$ and there is a single consumption good as well as a single risky asset in the economy, whose payoff at the end of the period is described by a random variable $\widetilde{x}$. We let $p$ denote the unit price of the risky asset in terms of consumption good, which means that both agents can sell their property rights on the risky asset against the delivery of the sure quantity $p$ at the end of the period. We assume that the agents have the same endowment, which consists of a half unit of the risky asset. The difference with the standard model stems from the fact that agents have possibly incorrect beliefs. We assume that the set of possible beliefs for agent 1 (agent 2) is parametrized by $\gamma$ (by $\theta)$ in a given set $B$. Our agents then have different probability measures over $(\Omega, F)$ respectively denoted by $Q_{\gamma}$ and $Q_{\theta}$. We denote by $E^{\gamma}$ and $E^{\theta}$ the expectation operators respectively associated to $Q_{\gamma}$ and $Q_{\theta}$. For a given random consumption $\widetilde{c}$, the expected utility of agent 1 (agent 2) is then given by $E^{\gamma}\left[u_{1}(\widetilde{c})\right]$ (by $E^{\theta}\left[u_{2}(\widetilde{c})\right]$ ). As in the standard portfolio problem, agents determine the optimal composition of their portfolio, in other words their optimal exposure to the risk.

Definition 1 A Walras equilibrium $\left(p ;\left(\alpha_{1}^{*}, \alpha_{2}^{*}\right)\right)$ is defined by a price $p$ and quantities $\left(\alpha_{1}^{*}, \alpha_{2}^{*}\right)$ of risky asset for each agent such that the quantity $\alpha_{i}^{*}$ is optimal for agent $i$ under her budget constraint, i.e.

$$
\begin{aligned}
& \alpha_{1}^{*}=\arg \max _{\alpha} E^{\gamma}\left[u_{1}\left(\frac{1}{2} p+\alpha(\widetilde{x}-p)\right)\right] \\
& \alpha_{2}^{*}=\arg \max _{\alpha} E^{\theta}\left[u_{2}\left(\frac{1}{2} p+\alpha(\widetilde{x}-p)\right)\right]
\end{aligned}
$$

and such that markets clear, i.e. $\alpha_{1}^{*}+\alpha_{2}^{*}=1$. 
The structure of the underlying Walras equilibrium problem is quite general and may be applied to several equilibrium problems in which heterogeneous risk-averse agents have to choose their optimal exposure to a given risk. For instance, when an insurance company and a reinsurance company have to determine an optimal retention rate.

In the next we will denote by $U_{1}(\gamma, \theta)$ and $U_{2}(\gamma, \theta)$ the ex-post utility levels of agent 1 and 2 at the Walras equilibrium, i.e.

$$
\begin{aligned}
& U_{1}(\gamma, \theta)=E\left[u_{1}\left(\frac{1}{2} p+\alpha_{1}^{*}(\widetilde{x}-p)\right)\right] \\
& U_{2}(\gamma, \theta)=E\left[u_{2}\left(\frac{1}{2} p+\alpha_{2}^{*}(\widetilde{x}-p)\right)\right]
\end{aligned}
$$

It may seem puzzling that the expectation is taken under the objective probability while we are assuming on the other hand that the agents have subjective beliefs. In fact, agents experience their utility while their beliefs may result from various sources (learning, strategic interactions, information,...). We could think of $U_{i}(\gamma, \theta)$ as the outcome of a process where the agents repeatedly face the same situation and where, each time, a different value of $\widetilde{x}$ is chosen by Nature according to the objective law of $\widetilde{x}$. If the number of repetitions is large enough, the average utility level obtained by each agent converges to the expected utility under the objective probability even though the agent is not conscious of this objective probability.

\subsection{The evolutionary framework}

Since equilibrium utility levels $U_{1}(\gamma, \theta)$ and $U_{2}(\gamma, \theta)$ depend upon agents' beliefs, the agents may (consciously or not) modify their beliefs to take advantage of this impact. For example, it may be beneficial for an agent, who is risk-tolerant, hence willing to be quite highly exposed to the risk, or equivalently interested in buying a high quantity of the risky asset, to underestimate the asset in order to benefit from a lower price.

To assess the evolutionary viability of the different possible beliefs, our model is embedded in a continuous time setting with two large populations of individuals, one for each player, and with a continuum of beliefs for each group. Each individual in the first (second) population corresponds to a possible belief for agent 1 (agent 2). We denote by $\Upsilon_{0}$ the initial distribution (over $B$ ) of beliefs in group 1 and by $\Theta_{0}$ the initial distribution (over $B$ ) of beliefs in group 2. In fact, these distributions correspond to the frequencies at which agent 1 (agent 2) selects the different beliefs. Both distributions are assumed to have full support over $B$ and to belong to the set $\Delta(B)$ of Borel probability distributions over $B$. We may then model agents' interaction as follows: at each date, an individual $\gamma$ in one population is randomly matched with an individual $\theta$ of the other population leading to Walras equilibrium utility levels $U_{1}(\gamma, \theta)$ and $U_{2}(\gamma, \theta)$. Since we cast our analysis 
in an evolutionary setting, these equilibrium payoffs will represent fitness in the sense of evolutionary theory, that is to say the capability of an individual to reproduce. This formulation leads to a natural selection process among different beliefs in each population. At each date $t \geq 0$, the pair of populations is characterized by a pair of distributions $\left(\Upsilon_{t}, \Theta_{t}\right) \in \Delta(B) \times \Delta(B)$ of $(\gamma, \theta)$. The average fitness levels of the individuals of types $\gamma$ and $\theta$ are then given by $\int U_{1}(\gamma, \theta) d \Theta_{t}$ and $\int U_{2}(\gamma, \theta) d \Upsilon_{t}$.

We assume that the selection dynamics are monotonically increasing in average fitness. That is, we assume that the distributions of types evolve as follows:

$$
\begin{aligned}
\frac{d}{d t} \Upsilon_{t}(A) & =\int_{A} V_{1}\left(\gamma, \Theta_{t}\right) d \Upsilon_{t}, & & A \subset B, \text { Borel measurable, } \\
\frac{d}{d t} \Theta_{t}(A) & =\int_{A} V_{2}\left(\Upsilon_{t}, \theta\right) d \Theta_{t}, & & A \subset B, \text { Borel measurable, }
\end{aligned}
$$

where $V_{1}$ and $V_{2}$ are continuous ${ }^{6}$ growth-rate functions that satisfy

$$
\begin{aligned}
& V_{1}\left(\gamma, \Theta_{t}\right)>V_{1}\left(\tilde{\gamma}, \Theta_{t}\right) \Longleftrightarrow \int U_{1}(\gamma, \theta) d \Theta_{t}>\int U_{1}(\tilde{\gamma}, \theta) d \Theta_{t} \\
& V_{2}\left(\Upsilon_{t}, \theta\right)>V_{2}\left(\Upsilon_{t}, \tilde{\theta}\right) \Longleftrightarrow \int U_{2}(\gamma, \theta) d \Upsilon_{t}>\int U_{2}(\gamma, \theta) d \Upsilon_{t}
\end{aligned}
$$

To ensure that $\Upsilon_{t}$ and $\Theta_{t}$ remain probability measures for each $t$, we also assume that $V_{1}$ and $V_{2}$ satisfy

$$
\int V_{1}\left(\gamma, \Theta_{t}\right) d \Upsilon_{t}=0 \quad \text { and } \quad \int V_{2}\left(\Upsilon_{t}, \theta\right) d \Theta_{t}=0 \quad \text { for each } t
$$

Note that (1) and (2) may be rewritten as follows

$$
\frac{d}{d t} \ln \Upsilon_{t}(A)=\frac{\int_{A} V_{1}\left(\gamma, \Theta_{t}\right) d \Upsilon_{t}}{\Upsilon_{t}(A)}, \quad \frac{d}{d t} \ln \Theta_{t}(A)=\frac{\int_{A} V_{2}\left(\Upsilon_{t}, \theta\right) d \Theta_{t}}{\Theta_{t}(A)}
$$

which means that the growth rate of $\Upsilon_{t}(A)$ is given by the average value of $V_{1}\left(\gamma, \Theta_{t}\right)$ over the set $A$.

This dynamics is similar to the dynamics considered in Heifetz et al. (2007a,b) and generalizes the replicator dynamics (see e.g. Cressman et al., 2006) where the functions $V_{i}$ are given by

$$
\begin{aligned}
V_{1}\left(\gamma, \Theta_{t}\right) & =\int U_{1}(\gamma, \theta) d \Theta_{t}-\int U_{1}(\gamma, \theta) d \Theta_{t} d \Upsilon_{t} \\
V_{2}\left(\gamma, \Theta_{t}\right) & =\int U_{2}(\gamma, \theta) d \Upsilon_{t}-\int U_{2}(\gamma, \theta) d \Theta_{t} d \Upsilon_{t} .
\end{aligned}
$$

\footnotetext{
${ }^{6} V_{1}$ and $V_{2}$ are respectively defined over $B \times \Delta(B)$ and $\Delta(B) \times B$. The continuity property is meant here in the sense of the product of the topology of $B$ and of the weak-topology of $\Delta(B)$.
} 
Equations (1) to (4) reflect the idea that the proportion of more successful types in the population increases from one period to another at the expense of less successful types (in the replicator dynamics, the growth rate of a given type is directly proportional to its success measured by the difference between its payoff and the average payoff). If the populations under consideration really correspond to different individuals with different types, then these equations may reflect the fact that more successful individuals will proliferate and that their descendants inherit their characteristics either genetically or by education. An alternative explanation is that more successful individuals are imitated more often. If the populations under consideration are interpreted as possible beliefs for agent 1 or agent 2 , the distributions we introduced correspond then to mixed strategies played by each agent. Without specific information about the objective value of $\gamma$ or $\theta$ (the values that correspond to the objective/rational belief), each agent tries different beliefs following a given distribution. Her distribution evolves then in order to give more weight to the beliefs that led to higher average payoffs at the expense of the beliefs that led to lower average payoffs. In this sense, each agent constructs her beliefs pragmatically. We refer to Heifetz et al. (2007a,b) and Oechssler and Riedel (2001) for the technical conditions that guarantee that the system of equations (1)-(2) has a well defined solution.

We are interested in the evolutionary characteristics of the beliefs in this dynamic problem. More precisely, we are interested in the following questions. Does this model of evolutionary strategic beliefs lead to subjectivity in beliefs? Does it generate heterogeneous beliefs? Is there a link between risk-tolerance and beliefs and what is the nature of this link? Is there a pessimistic/optimistic bias at the equilibrium at the individual as well as at the collective level? What are the consequences on the equilibrium characteristics and in particular on the risk premium?

\subsection{The underlying 2-player game}

Before going further in this direction, let us consider the game $\Gamma=\left(B, B, U_{1}, U_{2}\right)$ in which the strategic set of each agent is given by $B$ and the payoff functions are given by $U_{1}$ and $U_{2}$. The game $\Gamma$ corresponds to a game in which each agent chooses her belief in order to maximize her utility taking into account her own impact on equilibrium prices (and therefore on equilibrium utility levels).

Definition $2 A$ Nash equilibrium of the game $\Gamma$ is defined as a pair of beliefs $\left(\gamma^{*}, \theta^{*}\right) \in$ $B \times B$ such that

$$
\begin{aligned}
& U_{1}\left(\gamma^{*}, \theta^{*}\right) \geq U_{1}\left(\gamma, \theta^{*}\right) \text { for all } \gamma \in B \\
& U_{2}\left(\gamma^{*}, \theta^{*}\right) \geq U_{2}\left(\gamma^{*}, \theta\right) \text { for all } \theta \in B
\end{aligned}
$$


The Nash equilibrium in the game $\Gamma$ is the analogue of the notion of Nash equilibrium in demand schedules (introduced by Kyle, 1989) restricted to demand schedules of a specific form (namely, demand schedules that are parametrized by underlying beliefs and that correspond to the optimal demands of agents endowed with those beliefs).

Let us assume that the game $\Gamma$ admits a Nash equilibrium $\left(\gamma^{*}, \theta^{*}\right)$, by definition we have $U_{1}\left(\gamma^{*}, \theta^{*}\right) \geq U_{1}\left(\gamma, \theta^{*}\right)$ for all $\gamma \in B$ and then $V_{1}\left(\gamma^{*}, \delta_{\theta^{*}}\right) \geq V_{1}\left(\gamma, \delta_{\theta^{*}}\right)$ where $\delta_{\theta^{*}}$ is the Dirac measure at $\theta^{*}$. The average value of $V_{1}\left(\gamma, \delta_{\theta^{*}}\right)$ over $\left\{\gamma^{*}\right\}$ is then higher than the average value of $V_{1}\left(\gamma, \delta_{\theta^{*}}\right)$ over any set $A \subset B$. This means that when $\Theta_{t}$ is kept constant equal to $\delta_{\theta^{*}}$ then the selection dynamics described by (1) will favor the strategy $\gamma^{*}$. Conversely, when $\Upsilon_{t}$ is kept constant equal to $\delta_{\gamma^{*}}$ then the selection dynamics described by (2) will favor the strategy $\theta^{*}$. The Nash equilibria of the game $\Gamma$ are then good candidates for the asymptotic behaviour of the selection dynamics described by (1) and (2).

These links between our evolutionary problem and the game $\Gamma$ will reveal to be useful in order to analyze the properties of the equilibrium characteristics.

\section{Exponential utility and normal distributions set- ting}

For analytical tractability, we first consider exponential utility functions with normal distributions and provide explicit results.

Agents have utility functions for consumption of the form $u_{1}(c)=-\exp \left(-\frac{c}{\theta_{1}}\right)$ and $u_{2}(c)=-\exp \left(-\frac{c}{\theta_{2}}\right)$, where $\theta_{i}>0$ denotes the degree of (absolute) risk-tolerance of agent $i$. Moreover, we assume that $\widetilde{x}$ is normally distributed, with mean $\mu$ and variance $\sigma^{2}$.

Let us first determine the Walras equilibrium characteristics ${ }^{7}$ when agent 1 believes that $\widetilde{x}$ is normal with mean $\mu_{1}$ and variance $\sigma^{2}$ and agent 2 believes that $\widetilde{x}$ is normal with mean $\mu_{2}$ and variance $\sigma^{2}$. The parameters $\mu_{1}$ and $\mu_{2}$ play then the role of the parameters $\gamma$ and $\theta$ of the previous section and are assumed to be drawn from a set $B=[\underline{\mu}, \bar{\mu}]$ of possible beliefs that contains the objective value $\mu$.

In such a setting and for a given $p$, the optimal demand $\alpha_{i}(p)$ of agent $i$ is given by $\alpha_{i}(p)=\theta_{i} \frac{\mu_{i}-p}{\sigma^{2}}$. The market clearing condition $\alpha_{1}(p)+\alpha_{2}(p)=1$ imposes then that the equilibrium price $p\left(\mu_{1}, \mu_{2}\right)$ of the risky asset is given by

$$
p\left(\mu_{1}, \mu_{2}\right)=\frac{\theta_{1}}{\theta_{1}+\theta_{2}} \mu_{1}+\frac{\theta_{2}}{\theta_{1}+\theta_{2}} \mu_{2}-\frac{\sigma^{2}}{\theta_{1}+\theta_{2}}
$$

\footnotetext{
${ }^{7}$ Walrasian equilibrium models with heterogeneous beliefs have been studied, among others, by Williams (1977), Abel (1989), Detemple-Murthy (1994), Calvet et al. (2002) and Jouini-Napp (2006, 2007).
} 
which is the equilibrium price in an economy in which agents share the same expectations given by $\frac{\theta_{1} \mu_{1}+\theta_{2} \mu_{2}}{\theta_{1}+\theta_{2}}$. In other words, the Walrasian equilibrium price in an economy with heterogeneous beliefs is the equilibrium price in an economy in which the belief of the representative agent (whose risk-tolerance $\bar{\theta}$ is given, as in the standard setting, by the sum of the individual risk-tolerances) is given by the average of the heterogeneous beliefs, weighted by the risk-tolerance. Moreover, replacing $p$ by the expression of the equilibrium price, we obtain that the optimal demand $\alpha_{i}^{*}$ of agent $i$ at the equilibrium is given by

$$
\alpha_{i}^{*}\left(\mu_{1}, \mu_{2}\right)=\alpha_{i}\left(p\left(\mu_{1}, \mu_{2}\right), \mu_{i}\right)=\frac{\theta_{i}}{\bar{\theta}}\left[1+\theta_{j} \frac{\mu_{i}-\mu_{j}}{\sigma^{2}}\right]
$$

and the part of the risk borne by agent $i$ depends upon both his level of risk-tolerance and his belief.

The resulting utility levels are then given by

$$
U_{i}\left(\mu_{1}, \mu_{2}\right)=E\left[u_{i}\left\{\frac{1}{2} p\left(\mu_{i}, \mu_{j}\right)+\left\{\widetilde{x}-p\left(\mu_{i}, \mu_{j}\right)\right\} \alpha_{i}^{*}\left(\mu_{i}, \mu_{j}\right)\right\}\right], \quad i=1,2 .
$$

Letting $R P$ (resp. $R P^{s t d d}$ ) denote the risk premium $\mu-p$ in this setting (resp. in the standard setting) we obtain

$$
R P=\frac{\sigma^{2}}{\bar{\theta}}+\left(\mu-\frac{\theta_{1} \mu_{1}+\theta_{2} \mu_{2}}{\theta_{1}+\theta_{2}}\right)=R P^{s t d d}+\left(\mu-\frac{\theta_{1} \mu_{1}+\theta_{2} \mu_{2}}{\theta_{1}+\theta_{2}}\right)
$$

which means that the risk premium in an economy with heterogeneous subjective beliefs is higher than in the standard rational expectations setting if and only if the belief of the representative agent, which is the risk tolerance weighted average of the individual beliefs, is pessimistic, where pessimistic is meant in the sense that the mean of the risky asset's payoff is underestimated. It is therefore particularly interesting to explore when and why individuals are pessimistic, as well as the nature of the link between risk tolerance and pessimism. In the present paper, the individual beliefs are determined endogenously and we analyze their properties, especially in terms of pessimism, correlation between pessimism and risk tolerance and impact on the risk premium.

With these specifications and for given initial distributions $\Upsilon_{0}$ and $\Theta_{0}$ with full support over $B$, the selection dynamics given by (1) and (2) is fully described. The game $\Gamma=$ $\left(B, B, U_{1}, U_{2}\right)$ is also fully described.

Proposition 3 Let us consider a model with two agents, exponential utility functions and a risky asset $\widetilde{x} \sim \mathcal{N}\left(\mu, \sigma^{2}\right)$ and let us assume that the possible beliefs are of the form $\widetilde{x} \sim \mathcal{N}\left(\mu^{\prime}, \sigma^{2}\right)$ with $\mu^{\prime} \in B=[\mu, \bar{\mu}]$. We have

1. For given initial distributions $\Upsilon_{0}$ and $\Theta_{0}$ in $\Delta(B)$ with full support over $B$, the evolutionary process converges to a pair of Dirac distributions $\left(\delta_{\hat{\mu}_{1}}, \delta_{\hat{\mu}_{2}}\right)$ where $\left(\widehat{\mu}_{1}, \widehat{\mu}_{2}\right)$ 
is the unique Nash equilibrium of the game $\Gamma=\left(B, B, U_{1}, U_{2}\right)$.

2. The surviving beliefs $\left(\widehat{\mu}_{1}, \widehat{\mu}_{2}\right)$ are given by

$$
\widehat{\mu}_{1}=\mu-\frac{\sigma^{2}}{4 \theta_{2}\left(\theta_{1}+\theta_{2}\right)}\left(\theta_{1}-\theta_{2}\right), \quad \widehat{\mu}_{2}=\mu-\frac{\sigma^{2}}{4 \theta_{1}\left(\theta_{1}+\theta_{2}\right)}\left(\theta_{2}-\theta_{1}\right) .
$$

Asymptotically, the more risk-tolerant agent is pessimistic, in the sense that he behaves as if the mean of $\widetilde{x}$ lied below its true value, and the less risk-tolerant agent is optimistic. Moreover, the more risk-tolerant agent is more pessimistic than the less risk-tolerant agent is optimistic and the unweighted average of the beliefs is pessimistic:

$$
\frac{\widehat{\mu}_{1}+\widehat{\mu}_{2}}{2}=\mu-\frac{1}{8} \frac{\left(\theta_{1}-\theta_{2}\right)^{2} \sigma^{2}}{\theta_{1} \theta_{2} \bar{\theta}} .
$$

3. Asymptotically, the representative agent is pessimistic, i.e. the average of the individual beliefs weighted by the risk-tolerance is pessimistic. More precisely,

$$
\frac{\theta_{1} \widehat{\mu}_{1}+\theta_{2} \widehat{\mu}_{2}}{\theta_{1}+\theta_{2}}=\mu-\frac{1}{4} \frac{\left(\theta_{1}-\theta_{2}\right)^{2} \sigma^{2}}{\theta_{1} \theta_{2}\left(\theta_{1}+\theta_{2}\right)} \text {. }
$$

4. Asymptotically, the risk premium RP (resp. the price) is higher (resp. lower) than in the standard rational expectations equilibrium. More precisely

$$
R P=R P^{s t d d}+\left(\mu-\frac{\theta_{1} \widehat{\mu}_{1}+\theta_{2} \widehat{\mu}_{2}}{\theta_{1}+\theta_{2}}\right)=R P^{s t d d}+\frac{1}{4} \frac{\left(\theta_{1}-\theta_{2}\right)^{2} \sigma^{2}}{\theta_{1} \theta_{2}\left(\theta_{1}+\theta_{2}\right)} .
$$

5. Asymptotically, the optimal demands are given by

$$
\alpha_{1}^{*}=\frac{\theta_{1}}{\theta_{1}+\theta_{2}}+\frac{\left(\theta_{2}-\theta_{1}\right)}{4\left(\theta_{1}+\theta_{2}\right)}, \quad \alpha_{2}^{*}=\frac{\theta_{2}}{\theta_{1}+\theta_{2}}+\frac{\left(\theta_{1}-\theta_{2}\right)}{4\left(\theta_{1}+\theta_{2}\right)} .
$$

which means that the volumes of trade (and the risk sharing) are reduced compared to the standard setting. The more risk tolerant (resp. risk averse) agent selects a less (resp. more) risky portfolio.

6. If $\frac{2}{3} \theta_{1} \leq \theta_{2} \leq \frac{3}{2} \theta_{1}$, then, at the equilibrium, both agents asymptotically have utility levels that are lower than in the Walrasian setting. Otherwise, the utility level of the more risk tolerant agent increases (with respect to the Walrasian setting) while the utility level of the more risk averse agent decreases.

Evolution selects then only one belief for each group of agents and the pair of asymptotic beliefs corresponds to the unique Nash equilibrium of the game $\Gamma$. Therefore, the surviving belief for a given agent corresponds to the belief that would be chosen by the agent under consideration in a model where such an agent would take into account 
her impact on equilibrium prices (and therefore on equilibrium utility levels) and would choose her beliefs accordingly. In this sense, our beliefs are strategic ones. They correspond to the optimal strategy for a strategic agent whose strategic variable is given by her belief.

Note that our construction of evolutionary strategic beliefs leads to subjective and heterogeneous beliefs. Indeed, evolutionary strategic beliefs differ from the objective belief, agents 1 and 2 differ in their beliefs and, as expressed in Equations (9), belief heterogeneity takes its roots in the difference in risk-aversion levels. Besides, more than just being "heterogeneous", evolutionary strategic beliefs are "antagonistic" in the sense that one of the agents is optimistic $\left(\widehat{\mu}_{i}>\mu\right)$ and the other one is pessimistic $\left(\widehat{\mu}_{i}<\mu\right)$.

With the strategic interpretation, the different qualitative results are easy to interpret.

The pessimism of the more risk-tolerant agent can be interpreted as follows. Suppose that agent 1 is more risk-tolerant. At the equilibrium, since agents initially only differ in their level of risk-aversion, the risky asset's demand for agent 1 is positive. His expected utility from trade is then decreasing in the price of the risky asset. The choice of a pessimistic belief is associated to a lower demand, hence to a lower price and a higher expected utility. The evolutionary strategic belief balances this benefit of pessimism against the costs of worse decision making. The converse reasoning applies to agent 2 , who, at the equilibrium, has a negative demand in the risky asset and benefits from optimism. Another way to interpret the pessimism of the more risk-tolerant agent is to analyze the situation in the neighborhood of the objective belief and the associated equilibrium, i.e. the Walras equilibrium with objective belief. Indeed, a deviation from the objective belief has potentially two effects on the utility level: a quantity effect and a price effect. The quantity effect is equal to zero due to the optimal quantity choice condition in the Walras equilibrium and the price effect is positive for the less risktolerant agent (i.e. the agent that has a negative net demand) and is negative for the more risk-tolerant agent.

As a consequence of the positive correlation between pessimism (optimism) and risktolerance (risk-aversion), the more risk-tolerant will insure the less risk-tolerant less than in the standard setting, which induces less risk-sharing.

The average (unweighted) belief is pessimistic, which means that the risk-tolerant agent is more pessimistic than the risk-averse is optimistic. This result can be understood as follows. As we have seen, the evolutionary strategic belief results from an arbitrage between the benefit of a low price induced by pessimism for the risk-tolerant (resp. the benefit of a high price induced by optimism for the risk-averse) against the costs of worse decision making. Let us explore this point further. At the Walras equilibrium, the marginal utility of the less risk-tolerant agent associated to a marginal increase of $\mu$ is positive and equal to the marginal utility of the more risk-tolerant agent associated to a marginal decrease of $\mu$. By definition, the evolutionary strategic beliefs correspond to zero 
marginal utilities. When the more risk-tolerant agent becomes more pessimistic and when the less risk-tolerant agent becomes more optimistic, their marginal utilities decrease at different rates. The difference between these two rates originates in the variance terms in the utility functions and more precisely in the terms $\left(\Delta \alpha_{1}\right)^{2}$ and $\left(\Delta \alpha_{2}\right)^{2}$. Due to the market clearing condition, these two terms are equal; however, by definition of the risktolerance coefficient, they are weighted respectively by $\frac{1}{\theta_{1}^{2}}$ and $\frac{1}{\theta_{2}^{2}}$ in the utility functions. This leads to a slower decrease of the marginal utility for the more risk-tolerant agent and then to a more pronounced divergence from the objective belief for that agent.

The consensus belief, which is given by the average of the individual beliefs weighted by the risk-tolerance, is then obviously pessimistic. Intuitively, the more risk-tolerant agents make the market, and the consensus belief reflects the characteristics of the more risk-tolerant. Since we have just seen that the more risk-tolerant is pessimistic, it is consistent to obtain a pessimistic consensus belief.

The risk premium is greater than in the standard rational expectations equilibrium, which is interesting in light of the risk premium puzzle. This is easily understandable, since, as we have seen, in equilibrium models with heterogeneous beliefs the risk premium is higher than in the standard setting if and only if the consensus belief is pessimistic. The reason why pessimism increases the risk premium is not that a pessimistic representative agent requires a higher risk premium. He requires the same risk premium but his pessimism leads him to underestimate the average rate of return of the risky asset. Thus the objective expectation of the equilibrium risk premium is greater than the representative agent's subjective expectation, hence is greater than the standard risk premium (see Abel, 2002, and Jouini-Napp, 2006).

To sum up, our construction of endogenous beliefs through an evolutionary strategic approach leads to beliefs that are different from the objective belief, heterogeneous, and antagonistic (one is optimistic and the other is pessimistic). There is a positive correlation between risk-tolerance (resp. risk-aversion) and pessimism (resp. optimism), which leads to less risk-sharing and to a higher risk premium.

Our results are robust to variations in the initial endowments as long as the more risk-tolerant agent has a positive net demand, i.e. as long as the more risk-tolerant agent insures the less risk-tolerant one, which is a natural situation. At first sight, a negative supply in the risky asset seems to lead to an optimistic bias. Indeed, in that case, the strategic behaviour induces an upward bias on the mean of the risky asset distribution but this corresponds to a pessimistic bias on the total wealth of the economy. The unique situation where all the effects we exhibited disappear corresponds to the case where there is no aggregate risk (i.e. when the total supply in risky assets is equal to zero). Indeed, in such a framework, there is no trade at the Walrasian equilibrium and there is then no price effect and no utility gain associated to a deviation from the objective belief. This is natural and still in line with our conclusions since, in such a setting without aggregate 
risk, the concepts of optimism and pessimism do not have any meaning.

\section{Strategic vs "optimal" beliefs}

The construction of endogenous subjective beliefs that are solutions of a given utility maximization problem has been considered in recent literature by Brunnermeier-Parker (2005), Gollier (2005), Gollier-Muermann (2010), Brunnermeier et al. (2007). In our framework, the subjective beliefs are not only optimal but strategic. Indeed, they do not result from an individual utility maximization problem but from an evolutionary process or equivalently from a Nash equilibrium in which each agent takes into account the impact of his choices on the equilibrium price and allocations. As we shall analyze it in detail, in a non-strategic setting where agents choose their belief in order to maximize a criterion related to their well-being, it is immediate that the optimal belief must be optimistic for all agents and that all agents select a riskier portfolio. In our setting, there is no such immediate intuition for a given systematic bias.

Let us compare our results with those that are obtained in an optimal non-strategic framework. More precisely, adopting the same framework and notations as above, we consider the following concept of optimal beliefs, which corresponds to a simplified version of Brunnermeier-Parker (2005), Gollier (2005) and Brunnermeier et al. (2007).

Definition 4 For a given price $p$, an optimal (non-strategic) belief $\check{\mu}_{i}(p)$ for agent $i$ is defined as the solution of

$$
\arg \max _{\mu_{i} \in[\underline{\mu}, \bar{\mu}]} E_{i}\left[u_{i}\left(\frac{1}{2} p+\alpha_{i}\left(p, \mu_{i}\right)(\widetilde{x}-p)\right)\right]
$$

where $E_{i}$ is the expectation operator associated ${ }^{8}$ to the belief $\mu_{i}$.

The belief $\check{\mu}_{i}(p)$ is optimal in the sense that it maximizes over the set $[\mu, \bar{\mu}]$ the well-being of agent $i$.

The original definition of optimal beliefs introduced by Brunnermeier-Parker (2005) and further studied by Brunnermeier et al. (2007) and Gollier (2005) considers a weighted average of the objectively expected utility and of the subjectively expected utility. Our definition is more simple but it is easy to check that the results obtained below, under Definition 4, remain valid under the original definition. This will be further discussed at the end of this section.

We can now define an associated equilibrium concept as follows.

\footnotetext{
${ }^{8}$ More precisely, $E_{i}$ is the expectation operator associated to a probability $P_{i}$ that represents agents $i^{\prime}$ s belief and under which $\widetilde{x} \sim \mathcal{N}\left(\mu_{i}, \sigma^{2}\right)$.
} 
Definition 5 An equilibrium price with optimal (non-strategic) beliefs is defined as a price $\check{p}$ such that agents have optimal demands and optimal (non-strategic) beliefs and such that markets clear, i.e.

$$
\alpha_{1}\left(\check{p}, \check{\mu}_{1}(\check{p})\right)+\alpha_{2}\left(\check{p}, \check{\mu}_{2}(\check{p})\right)=1 \text {. }
$$

Proposition 6 In the setting of the previous section (exponential utility and normal distributions), we have

1. For a given price $p$, the optimal (non-strategic) belief $\check{\mu}_{i}(p)$ for agent $i$ solves

$$
\max _{\mu \in\{\underline{\mu}, \bar{\mu}\}}(\mu-p)^{2}
$$

2. If $\frac{\sigma^{2}}{\theta_{1}+\theta_{2}} \geq \frac{\bar{\mu}-\underline{\mu}}{2}$, then the equilibrium is characterized by $\check{p}=\bar{\mu}-\frac{\sigma^{2}}{\theta_{1}+\theta_{2}}$ and $\check{\mu}_{1}(\check{p})=$ $\check{\mu}_{2}(\check{p})=\bar{\mu}$. The agents share the same optimistic belief and the risk premium is lower than in the standard setting.

3. If $\frac{\theta_{1} \underline{\mu}+\theta_{2} \bar{\mu}}{\theta_{1}+\theta_{2}}-\frac{\sigma^{2}}{\theta_{1}+\theta_{2}}=\frac{\mu+\bar{\mu}}{2}$, where $\theta_{1}<\theta_{2}$, then the equilibrium is characterized by $\check{p}=$ $\frac{\underline{\underline{\mu}}+\bar{\mu}}{2}, \check{\mu}_{1}(\check{p})=\mu$ and $\check{\mu}_{2}(\check{p})=\bar{\mu}$. The more risk-tolerant agent is the more optimistic and the consensus belief $\frac{\theta_{1} \underline{\mu}+\theta_{2} \bar{\mu}}{\theta_{1}+\theta_{2}}$ is more optimistic than the equally weighted belief $\frac{\mu+\bar{\mu}}{2}$.

We have then two possible situations but both of them induce an optimistic bias at the aggregate level. Furthermore, unless $\frac{\theta_{1} \mu+\theta_{2} \bar{\mu}}{\theta_{1}+\theta_{2}}-\frac{\sigma^{2}}{\theta_{1}+\theta_{2}}=\frac{\mu+\bar{\mu}}{2}$, there is no belief heterogeneity and both agents are optimistic. In fact, even when $\frac{\theta_{1} \underline{\mu}+\theta_{2} \bar{\mu}}{\theta_{1}+\theta_{2}}-\frac{\sigma^{2}}{\theta_{1}+\theta_{2}}=\frac{\mu+\bar{\mu}}{2}$, the more risk averse agent is not truly pessimistic. Indeed, it is easy to check that this agent is short in the risky asset and is then optimistic with respect to his own allocation, i.e. overestimates the return of his own portfolio.

Notice that for $\frac{\sigma^{2}}{\theta_{1}+\theta_{2}}<\frac{\bar{\mu}-\underline{\mu}}{2}$ and $\frac{\sigma^{2}}{\theta_{1}+\theta_{2}} \neq \frac{\mu+\bar{\mu}}{2}-\frac{\theta_{1} \underline{\mu}+\theta_{2} \bar{\mu}}{\theta_{1}+\theta_{2}}$ where agent 1 is less risktolerant than agent 2 , there is no equilibrium. A natural extension of the model would consist in allowing for mixed strategies or for a continuum of agents. We retrieve then an equilibrium in which a proportion $\alpha$ of the agents choose the belief $\underline{\mu}$ and a proportion $(1-\alpha)$ choose the belief $\bar{\mu}$. For instance, if we assume that the distribution of beliefs is independent of the distribution of risk-tolerances, the market clearing condition leads to

$$
\alpha \underline{\mu}+(1-\alpha) \bar{\mu}-\frac{\sigma^{2}}{\int \theta_{i} d i}=\frac{\underline{\mu}+\bar{\mu}}{2} .
$$

The proportion $\alpha$ is then perfectly determined if $\frac{\sigma^{2}}{\int \theta_{i} d i} \leq \frac{\bar{\mu}-\underline{\mu}}{2}$. The solution $\alpha$ is always lower than $\frac{1}{2}$ which means that the consensus belief is always optimistic. This equilibrium in which each agent is indifferent between two possible beliefs and in which the market 
clearing condition imposes the proportions of agents choosing each belief resembles the equilibrium obtained in Brunnermeier-Parker (2005).

These results are analogous to those of Brunnermeier-Parker (2005) even if in their case there is no aggregate risk $^{9}$.

We would obtain the same kind of results if we considered a weighted average of the objectively expected utility and the subjectively expected utility as in the original model of Brunnermeier-Parker (2005)

$$
\max _{\mu_{i} \in K}\left\{\beta E\left[u_{i}\left(\frac{1}{2} p+\alpha_{i}\left(p, \mu_{i}\right)(\widetilde{x}-p)\right)\right]+(1-\beta) E_{i}\left[u_{i}\left(\frac{1}{2} p+\alpha_{i}\left(p, \mu_{i}\right)(\widetilde{x}-p)\right)\right]\right\} .
$$

For $\beta$ large enough, in other words when the weight on the objective expectation is beyond a given threshold, then the agents share the same belief and this belief is optimistic. Otherwise, there is not a unique optimal belief, agents have extreme beliefs (i.e. $\underline{\mu}$ or $\bar{\mu}$ ), but the possible equilibria still lead to an optimistic average belief ${ }^{10}$. In all cases, the average optimal belief is optimistic leading to a lower risk premium. These results are similar to those obtained by Gollier (2005) in a general discrete distributions setting.

To conclude, in the optimal (non-strategic) setting, agents' beliefs are always optimistic (with respect to their own allocations) and the risk premium is always lower than in the rational expectations setting. Furthermore, except for specific degenerate situations (see equation 12), the agents share the same belief. The difference between optimal (non strategic) and strategic beliefs is now clear, since in the latter setting, there is belief heterogeneity, one agent is optimistic while the other is pessimistic and the risk premium is higher.

\section{The general case}

The purpose of this section is to analyze the robustness of the results of Section 3 to more general utility functions and distributions. In particular, we show that even with more general assumptions, the game $\Gamma$ associated to our evolutionary process admits Nash

\footnotetext{
${ }^{9}$ In this case, there is no absolute concept of optimism or pessimism and both agents are optimistic with respect to their own equilibrium allocation.

${ }^{10}$ More precisely, for $\beta<\frac{1}{2}$, the agents have extreme beliefs, $\underline{\mu}$ or $\bar{\mu}$, as above and there might exist equilibria with heterogeneous optimal beliefs if the model parameters satisfy a condition like equation (12). For $\beta>\frac{1}{2}$, i.e. when there is more weight on the objective expectation, and if $\bar{\mu}$ is sufficiently large $\left(\bar{\mu}>\bar{\mu}^{*}\right.$ for some $\left.\bar{\mu}^{*}\right)$ the agents share the same belief and this belief is an interior point of $[\underline{\mu}, \bar{\mu}]$. For $\beta=\frac{1}{2}$ or for $\bar{\mu} \leq \bar{\mu}^{*}$ the agents share the same belief $\bar{\mu}$.
} 
equilibria that have the same qualitative properties as those exhibited in the exponential/normal framework.

For this purpose, we consider a family of beliefs $\left(P_{\widetilde{x}}^{\mu}\right)_{\mu \in[\underline{\mu}, \bar{\mu}]}$, corresponding to the possible subjective distributions for $\tilde{x}$. We may assume without any loss of generality that the objective distribution corresponds to $\mu=0$. We further assume that $0 \in[\mu, \bar{\mu}]$, i.e. the objective distribution lies in the set of possible/plausible beliefs for the agents. In the next, we also assume that all the considered expectations exist and are finite. For $\mu \in[\underline{\mu}, \bar{\mu}]$, we let $f(., \mu)$ denote the density function of $P_{\widetilde{x}}^{\mu}$ with respect to the Lebesgue measure on $\mathbb{R}_{+}$and we let $E^{\mu}$ denote the expectation operator under the density $f(., \mu)$, i.e. $E^{\mu}[g(\widetilde{x})]=\int g(s) f(s, \mu) d x$. We will simply denote by $E$ (instead of $E^{0}$ ) the expectation operator associated to $P_{\widetilde{x}}^{0}$.

As in Section 3, our economy is composed of two agents, initially endowed with a half unit of the risky asset $\tilde{x}$ and we consider the game in which these agents can manipulate their beliefs and choose an optimal composition of their portfolio, taking into account the effect their trading has on price.

We make the following assumptions.

\section{Assumption (A)}

- The utility functions $u_{1}$ and $u_{2}$ are increasing, strictly concave and twice continuously differentiable on $\mathbb{R}_{+}$,

- Inada conditions: $u_{i}^{\prime}(0)=+\infty$ and $\lim _{x \rightarrow \infty} u_{i}^{\prime}(x)=0$,

- The family $\left(P_{\widetilde{x}}^{\mu}\right)_{\mu \in K}$ is increasing in the sense of the first-order stochastic dominance, i.e. for all $x>0$ we have $\int_{0}^{x} f(s, \mu) d s \geq \int_{0}^{x} f\left(s, \mu^{\prime}\right) d s$ for $\mu^{\prime} \geq \mu$ in $[\underline{\mu}, \bar{\mu}]$.

- The functions $s \mapsto s u_{i}^{\prime}(s), i=1,2$, are increasing.

The first condition is standard. The second one guarantees interior solutions to the individual portfolio choice problem. The third condition ensures an order on the set of admissible beliefs. The setting of Section 3 satisfies this condition. More generally, any family of beliefs $\left(P_{\widetilde{x}}^{\mu}\right)_{\mu \in \mathbb{R}_{+}}$such that $f(s, \mu)=g(s-\mu)$ for a given distribution function $g$ on $\mathbb{R}_{+}$satisfies this monotonicity condition. Another example is provided by a family of $\log$-normal distributions, $\left(\ln \mathcal{N}\left(\mu, \sigma^{2}\right)\right)_{\mu \in \mathbb{R}}$. The fourth condition guarantees that a firstorder stochastic dominance shift in the risky asset's payoffs increases the demand for the risky asset (see Gollier, 2001). The same portfolio property can be obtained without this condition if we replace the first-order stochastic dominance of the third condition by the monotone likelihood ratio order (Landsberger and Meilijson, 1990).

Let us first determine the Walras equilibrium characteristics. For a given belief $P_{\widetilde{x}}^{\mu}$ and for a given price $p$, the demand function of agent $i$ is given by

$$
\alpha_{i}(p, \mu)=\arg \max _{\alpha_{i}} E^{\mu}\left[u_{i}\left(\frac{1}{2} p+\alpha_{i}(\tilde{x}-p)\right)\right] .
$$


For a pair of beliefs $\left(\mu_{1}, \mu_{2}\right)$, the equilibrium price $p\left(\mu_{1}, \mu_{2}\right)$ is determined by the market-clearing condition $\alpha_{1}\left(p\left(\mu_{1}, \mu_{2}\right), \mu_{1}\right)+\alpha_{2}\left(p\left(\mu_{1}, \mu_{2}\right), \mu_{2}\right)=1$ and the associated optimal demand for agent $i$ is defined by $\alpha_{i}^{*}\left(\mu_{1}, \mu_{2}\right)=\alpha_{i}\left(p\left(\mu_{1}, \mu_{2}\right), \mu_{i}\right)$. The Walras equilibrium utility level for agent $i$ is then given by

$$
U_{i}\left(\mu_{1}, \mu_{2}\right)=E\left[u_{i}\left(\frac{1}{2} p\left(\mu_{1}, \mu_{2}\right)+\alpha_{i}^{*}\left(\mu_{1}, \mu_{2}\right)\left(\tilde{x}-p\left(\mu_{1}, \mu_{2}\right)\right)\right)\right] .
$$

Proposition 7 Under Assumption (A),

1. The functions $\alpha_{i}(p, \mu), p\left(\mu_{1}, \mu_{2}\right)$ and $\alpha_{i}^{*}\left(\mu_{1}, \mu_{2}\right)$ are well defined and satisfy $\frac{\partial \alpha_{i}}{\partial p}(p, \mu) \leq$ $0, \frac{\partial \alpha_{i}}{\partial \mu}(p, \mu) \geq 0, \frac{\partial p}{\partial \mu_{i}} \geq 0, \frac{\partial \alpha_{i}^{*}}{\partial \mu_{i}} \geq 0, \frac{\partial \alpha_{i}^{*}}{\partial \mu_{j}} \leq 0, i=1,2, j \neq i$.

2. If the game $\Gamma=\left(B, B, U_{1}, U_{2}\right)$, with $B=[\underline{\mu}, \bar{\mu}]$, admits an interior Nash equilibrium $\left(\hat{\mu}_{1}, \hat{\mu}_{2}\right)$, i.e. a Nash equilibrium such that $\hat{\mu}_{i} \in(\underline{\mu}, \bar{\mu})$, then one of the agents (agent $i$ ) is pessimistic and the other one (agent $j)$ is optimistic and we have $\alpha_{j}^{*}\left(\hat{\mu}_{1}, \hat{\mu}_{2}\right) \leq \frac{1}{2} \leq \alpha_{i}^{*}\left(\hat{\mu}_{1}, \hat{\mu}_{2}\right)$.

3. If one of the agents (say agent 1) is more risk-averse than the other one in the sense of Arrow-Pratt, then $\alpha_{1}^{*}\left(\hat{\mu}_{1}, \hat{\mu}_{2}\right) \leq \frac{1}{2}$, and there is a positive correlation between pessimism and risk-tolerance.

4. If the game $\Gamma=\left(B, B, U_{1}, U_{2}\right)$ admits a unique interior Nash equilibrium $\left(\hat{\mu}_{1}, \hat{\mu}_{2}\right)$ and if the functions $U_{1}$ and $U_{2}$ have increasing differences, i.e. $U_{i}\left(\mu_{1}^{\prime}, \mu_{2}^{\prime}\right)-U_{i}\left(\mu_{1}, \mu_{2}^{\prime}\right) \geq$ $U_{i}\left(\mu_{1}^{\prime}, \mu_{2}\right)-U_{i}\left(\mu_{1}, \mu_{2}\right)$ for all $\mu_{1}^{\prime} \geq \mu_{1}$ and $\mu_{2}^{\prime} \geq \mu_{2}$, then the evolutionary process defined by (1) and (2) converges to the pair of Dirac distributions $\left(\delta_{\hat{\mu}_{1}}, \delta_{\hat{\mu}_{2}}\right)$.

As far as the Walrasian equilibrium is concerned, we obtain first that the optimal demand of the agents (as a function of the price and the belief) increases with the belief and decreases with the price, which are natural properties. As a consequence, the equilibrium price increases with the beliefs, which is also natural; if the asset is more "desirable", its equilibrium price increases. An increase in the belief of agent $i$ has then two effects on his demand $\alpha_{i}^{*}$ at the equilibrium, a direct positive effect and an indirect negative effect due to the price increase. The global effect is positive. The effect of an increase of the belief of agent $i$ on the equilibrium demand $\alpha_{j}^{*}$ of the other agent is negative because there is only one effect, namely the price effect.

We obtain that the heterogeneity of strategic beliefs is robust to the choice of more general utility functions and distributions. Moreover, as in Section 3, one agent is optimistic and the other agent is pessimistic. The pessimistic agent is the one for which the net demand is positive. This result can be explained as before. For the agent who expresses a positive net demand for the risky asset, the choice of a pessimistic belief is associated to a lower price and a higher expected utility; the strategic belief balances this 
benefit of pessimism against the costs of worse decision making. The converse reasoning applies to the other agent, who, at the equilibrium, has a negative net demand in the risky asset and benefits from optimism.

The positive correlation between pessimism and risk-tolerance is also robust to this more general setting. When one of the agents is more risk-tolerant, his net demand is necessarily positive. Otherwise, he would have a negative demand which would lead to an optimistic belief while the other agent would be pessimistic, more risk-averse with a positive net demand. This is obviously impossible. The positive correlation follows.

These properties are then satisfied at any interior Nash equilibrium of the game $\Gamma=$ $\left(B, B, U_{1}, U_{2}\right)$ and, in particular, at any Nash equilibrium of the game $\Gamma^{\infty}=\left(\mathbb{R}, \mathbb{R}, U_{1}, U_{2}\right)$.

Additional assumptions about the structure of the game are needed in order to characterize the asymptotic behaviour of the evolutionary process. Exploring the conditions under which the game $\Gamma$ admits a unique Nash equilibrium or the conditions under which $U_{i}$ has increasing differences goes beyond the scope of this paper. However, if such conditions are satisfied then the evolutionary process converges to a pair of heterogeneous and antagonistic beliefs that are positively correlated with the level of risk aversion when the utility functions are well ordered with respect to this criterion.

\section{Extensions of the model}

In this section, we analyze the robustness of our results (heterogeneity of beliefs, positive correlation between pessimism and risk-tolerance in the game $\Gamma$, convergence of the evolutionary process to the Nash equilibrium of the game $\Gamma$ and, in the exponential utility/normal distributions setting, aggregate pessimism and higher risk premia) to other specifications of the model. In previous sections we analyzed the impact of strategic behaviour on beliefs when the set of possible beliefs is ordered for first-order stochastic dominance shifts. Such shifts correspond to changes on the mean for normal distributions and more generally can be interpreted in terms of optimism and pessimism. We can also be interested in the impact of strategic behaviour on beliefs when the set of possible beliefs is ordered for mean preserving spreads. Such spreads correspond to changes on the variance for normal distributions and more generally can be interpreted in terms of overconfidence and doubt/underconfidence. For tractability reasons, we will analyze this impact in an exponential utility and normal distributions framework (as in Section 3). We will also analyze, in an analogous framework, if our results are robust to the introduction of multiple sources of risk. 


\subsection{Disagreement on the variance}

The model is the same as in Section 3 except that the beliefs are now about the variance of $\widetilde{x}$. Our aim is to analyze how these distributions evolve through interactions between the 2 groups and through the evolutionary equations (1) and (2) introduced above. The payoff of the risky asset $\widetilde{x}$ is still normally distributed with mean $\mu$ and variance $\sigma^{2}$ and the set of beliefs is parametrized by $\sigma \in B \stackrel{\text { def }}{=}[\underline{\sigma}, \bar{\sigma}]$. With this parametrization, an agent who has a belief $\sigma_{i} \in B$ believes that $\widetilde{x}$ is normally distributed with mean $\mu$ and variance $\sigma_{i}^{2}$

The Walras equilibrium characteristics are determined as follows. If agent 1 has a belief $\sigma_{1}$ and agent 2 has a belief $\sigma_{2}$, then the optimal demand of agent $i$ is given by $\alpha_{i}\left(p, \sigma_{i}\right)=\theta_{i} \frac{\mu-p}{\sigma_{i}^{2}}$ and the market clearing price is given by $p\left(\sigma_{1}, \sigma_{2}\right)=\mu-\left(\frac{\theta_{1}}{\sigma_{1}^{2}}+\frac{\theta_{2}}{\sigma_{2}^{2}}\right)^{-1}$. The bias with respect to the objective belief can here be interpreted as a form of doubt/underconfidence $\left(\sigma_{i}>\sigma\right)$ or overconfidence $\left(\sigma_{i}<\sigma\right)$ instead of the pessimism/optimism biases $^{11}$ of Section 3 . Note that the obtained equilibrium price corresponds to the equilibrium price in an economy in which agents share the same belief, namely an harmonic average of the initial beliefs, weighted by the risk-tolerance. In other words, it is the equilibrium price in an economy in which the belief of the representative agent (whose risk-tolerance is given by $\bar{\theta}$, as in the standard setting) is given by the average of the initial beliefs, weighted by the risk-tolerance ${ }^{12}$. In this setting, the equilibrium risk premium is given by $\mu-p\left(\sigma_{1}, \sigma_{2}\right)=\left(\frac{\theta_{1}}{\sigma_{1}^{2}}+\frac{\theta_{2}}{\sigma_{2}^{2}}\right)^{-1}$, which means that the risk premium in an economy with heterogeneous subjective beliefs is higher than in the standard rational expectations setting if and only if the belief of the representative agent exhibits doubt (i.e., $\left.\left(\frac{\theta_{1}}{\sigma_{1}^{2}}+\frac{\theta_{2}}{\sigma_{2}^{2}}\right)^{-1}>\frac{\sigma^{2}}{\theta_{1}+\theta_{2}}\right)$. The Walras utility levels are then given by

$$
U_{i}\left(\sigma_{1}, \sigma_{2}\right)=E\left[u_{i}\left\{\frac{1}{2} p\left(\sigma_{1}, \sigma_{2}\right)+\left\{\widetilde{x}-p\left(\sigma_{1}, \sigma_{2}\right)\right\} \alpha_{i}^{*}\left(\sigma_{1}, \sigma_{2}\right)\right\}\right],
$$

where $\alpha_{i}^{*}\left(\sigma_{1}, \sigma_{2}\right)=\alpha_{i}\left(p\left(\sigma_{1}, \sigma_{2}\right), \sigma_{i}\right)$.

With these specifications and for given initial distributions $\Upsilon_{0}$ and $\Theta_{0}$ with full support over $B$, the selection dynamics given by (1) and (2) is fully described. The game $\Gamma=$ $\left(B, B, U_{1}, U_{2}\right)$ is also fully described.

Proposition 8 1. For any initial distributions $\Upsilon_{0}$ and $\Theta_{0}$ in $\Delta(B)$ with full support over $B$, the evolutionary process converges to a pair of Dirac distributions $\left(\delta_{\widehat{\sigma}_{1}}, \delta_{\widehat{\sigma}_{2}}\right)$

\footnotetext{
${ }^{11}$ See Abel (2002) for concepts of pessimism and doubt related to first and second order stochastic dominance.

${ }^{12}$ Walrasian equilibrium models in which agents have heterogeneous beliefs on the variance of the asset under consideration have been studied by, among others, Abel (1989, 2002), Duchin-Levy (2010), Fama-French (2007), Jouini-Napp (2006) and Yan (2010).
} 
where $\left(\widehat{\sigma}_{1}, \widehat{\sigma}_{2}\right)$ is the unique Nash equilibrium of the game $\Gamma=\left(B, B, U_{1}, U_{2}\right)$.

2. The asymptotic beliefs $\left(\widehat{\sigma}_{1}, \widehat{\sigma}_{2}\right)$ are given by

$$
\widehat{\sigma}_{1}^{2}=\sigma^{2}\left(1+\frac{\theta_{1}-\theta_{2}}{4 \theta_{2}}\right), \quad \widehat{\sigma}_{2}^{2}=\sigma^{2}\left(1+\frac{\theta_{2}-\theta_{1}}{4 \theta_{1}}\right)
$$

The more risk-tolerant agent exhibits doubt, in the sense that she overestimates the variance of $\widetilde{x}$, and the less risk-tolerant agent is overconfident. Moreover, the more risk-tolerant agent exhibits more doubt than the less risk-tolerant agent exhibits overconfidence, and the unweighted (harmonic) average of the beliefs exhibits doubt:

$$
2\left(\frac{1}{\widehat{\sigma}_{1}^{2}}+\frac{1}{\widehat{\sigma}_{2}^{2}}\right)^{-1}=\sigma^{2}\left(1+\frac{\left(\theta_{1}-\theta_{2}\right)^{2}}{2\left(\theta_{1}^{2}+\theta_{2}^{2}+6 \theta_{1} \theta_{2}\right)}\right)
$$

3. The representative agent exhibits doubt, i.e. the harmonic average of the individual beliefs weighted by the risk-tolerance exhibits doubt. More precisely,

$$
\left(\theta_{1}+\theta_{2}\right)\left(\frac{\theta_{1}}{\widehat{\sigma}_{1}^{2}}+\frac{\theta_{2}}{\widehat{\sigma}_{2}^{2}}\right)^{-1}=\sigma^{2}\left(1+\frac{3\left(\theta_{1}-\theta_{2}\right)^{2}}{16 \theta_{1} \theta_{2}}\right)
$$

4. The risk premium (resp. the price) is higher (resp. lower) than in the standard rational expectations equilibrium. More precisely

$$
R P=R P^{s t d d}+\left(\left(\frac{\theta_{1}}{\sigma_{1}^{2}}+\frac{\theta_{2}}{\sigma_{2}^{2}}\right)^{-1}-\frac{\sigma^{2}}{\theta_{1}+\theta_{2}}\right)=R P^{s t d d}+\frac{3\left(\theta_{1}-\theta_{2}\right)^{2}}{16 \theta_{1} \theta_{2}\left(\theta_{1}+\theta_{2}\right)} \sigma^{2} .
$$

We then retrieve the same properties as in Section 3 except that pessimism is replaced by doubt. Note that behavioral studies in a non strategic and non market context generally exhibit overconfidence instead of doubt. This bias is largely documented in the behavioral literature and in particular by Shiller (2000, p.142): "some basic tendency towards overconfidence appears to be a robust human character trait: the bias is definitely toward overconfidence rather than underconfidence". The strategic framework induces then an effect in the opposite direction and this might explain that Giordani and Söderlind (2006) "find little evidence of either overconfidence or doubt" in the survey of professional forecasters. Indeed, professional forecasters know that their forecasts have a direct as well as an indirect (their predictions influence the beliefs of many other investors) impact on prices and it is then natural for them to adopt a strategic behavior. 


\subsection{The model with two sources of risk}

The model is essentially the same as in Section 3 except that we now suppose that there are two sources of risk in the economy, whose associated payoffs at the end of the period are respectively denoted by $\widetilde{x}$ and $\widetilde{y}$. We let $p$ (resp. q) denote the price of $\widetilde{x}$ (resp. $\widetilde{y}$ ) and we assume that $\widetilde{x}$ and $\widetilde{y}$ are normally distributed, more precisely $\widetilde{x} \sim \mathcal{N}\left(\mu, \sigma^{2}\right)$ and $\widetilde{y} \sim \mathcal{N}\left(\nu, \varpi^{2}\right)$. We let $\rho$ denote the correlation between $\widetilde{x}$ and $\widetilde{y}$, i.e., $\rho \equiv \frac{\operatorname{cov}(\widetilde{x}, \widetilde{y})}{\sigma \varpi}$. Each agent is initially endowed with one half unit of each risky asset.

We assume that the set of possible beliefs is given by $B=[\underline{\mu}, \bar{\mu}] \times[\underline{\nu}, \bar{\nu}]$. For a given belief $\left(\mu_{i}, \nu_{i}\right)$ for agent $i$ and for a given price system $(p, q)$, it is immediate to show that the demand functions $\alpha_{i}$ and $\beta_{i}$ respectively in the first and the second asset are given by

$$
\begin{aligned}
\alpha_{i}\left(p,\left(\mu_{i}, \nu_{i}\right)\right) & =\theta_{i} \frac{\mu_{i}-p}{\sigma^{2}\left(1-\rho^{2}\right)}-\theta_{i} \frac{\left(\nu_{i}-q\right) \rho}{\sigma \varpi\left(1-\rho^{2}\right)} \\
\beta_{i}\left(p,\left(\mu_{i}, \nu_{i}\right)\right) & =\theta_{i} \frac{\nu_{i}-q}{\varpi^{2}\left(1-\rho^{2}\right)}-\theta_{i} \frac{\left(\mu_{i}-p\right) \rho}{\sigma \varpi\left(1-\rho^{2}\right)} .
\end{aligned}
$$

The Walras equilibrium price system $\left(p\left(\left(\mu_{1}, \nu_{1}\right),\left(\mu_{2}, \nu_{2}\right)\right), q\left(\left(\mu_{1}, \nu_{1}\right),\left(\mu_{2}, \nu_{2}\right)\right)\right)$ is determined by the market-clearing conditions $\alpha_{1}\left(p,\left(\mu_{1}, \nu_{1}\right)\right)+\alpha_{2}\left(p,\left(\mu_{2}, \nu_{2}\right)\right)=1$ and $\beta_{1}\left(p,\left(\mu_{1}, \nu_{1}\right)\right)+$ $\beta_{2}\left(p,\left(\mu_{2}, \nu_{2}\right)\right)=1$, which leads to an equilibrium price for the first (second) asset that only depends on agents' beliefs about that asset

$$
\begin{aligned}
& p\left(\mu_{1}, \mu_{2}\right)=\frac{\theta_{1} \mu_{1}+\theta_{2} \mu_{2}}{\bar{\theta}}-\frac{\sigma^{2}+\sigma \varpi \rho}{\bar{\theta}} \\
& q\left(\nu_{1}, \nu_{2}\right)=\frac{\theta_{1} \nu_{1}+\theta_{2} \nu_{2}}{\bar{\theta}}-\frac{\varpi^{2}+\sigma \varpi \rho}{\bar{\theta}}
\end{aligned}
$$

and to optimal quantities of assets at the equilibrium given by

$$
\begin{aligned}
& \alpha_{i}^{*}\left(\left(\mu_{1}, \nu_{1}\right),\left(\mu_{2}, \nu_{2}\right)\right)=\frac{\theta_{i}}{\bar{\theta}}\left[1+\frac{\theta_{j}\left(\mu_{i}-\mu_{j}\right)}{\sigma^{2}\left(1-\rho^{2}\right)}-\frac{\theta_{j}\left(\nu_{1}-\nu_{2}\right)}{\sigma \varpi\left(1-\rho^{2}\right)}\right] \\
& \beta_{i}^{*}\left(\left(\mu_{1}, \nu_{1}\right),\left(\mu_{2}, \nu_{2}\right)\right)=\frac{\theta_{i}}{\bar{\theta}}\left[1+\frac{\theta_{j}\left(\nu_{i}-\nu_{j}\right)}{\varpi^{2}\left(1-\rho^{2}\right)}-\frac{\theta_{j}\left(\mu_{i}-\mu_{j}\right)}{\sigma \varpi\left(1-\rho^{2}\right)}\right] .
\end{aligned}
$$

The Walras equilibrium utility level for agent $i$ is then given by

$U_{i}\left(\left(\mu_{1}, \nu_{1}\right),\left(\mu_{2}, \nu_{2}\right)\right)=E\left[u_{i}\left(\frac{1}{2} p\left(\mu_{1}, \mu_{2}\right)+\frac{1}{2} q\left(\nu_{1}, \nu_{2}\right)+\alpha_{i}^{*}\left(\tilde{x}-p\left(\mu_{1}, \mu_{2}\right)\right)+\beta_{i}^{*}\left(\widetilde{y}-q\left(\nu_{1}, \nu_{2}\right)\right)\right)\right]$

Proposition 9 1. For any initial distributions $\Upsilon_{0}$ and $\Theta_{0}$ in $\Delta(B)$ with full support over $B$, the evolutionary process converges to a pair of Dirac distributions $\left(\delta_{\left(\widehat{\mu}_{1}, \widehat{\nu}_{1}\right)}, \delta_{\left(\widehat{\mu}_{2}, \widehat{\nu}_{2}\right)}\right)$ where $\left(\left(\widehat{\mu}_{1}, \widehat{\nu}_{1}\right),\left(\widehat{\mu}_{2}, \widehat{\nu}_{2}\right)\right)$ is the unique Nash equilibrium of the 
game $\Gamma=\left(B, B, U_{1}, U_{2}\right)$.

2. The beliefs $\left(\widehat{\mu}_{i}, \widehat{\nu}_{i}\right)_{i=1,2}$ are given by

$$
\widehat{\mu}_{i}=\mu-\frac{\left(\theta_{i}-\theta_{j}\right)\left(\sigma^{2}+\sigma \varpi \rho\right)}{4 \theta_{j} \bar{\theta}}, \quad \widehat{\nu}_{i}=\nu-\frac{\left(\theta_{i}-\theta_{j}\right)\left(\varpi^{2}+\sigma \varpi \rho\right)}{4 \theta_{j} \bar{\theta}}
$$

3. The risk premium (resp. the price) is higher (resp. lower) than in the standard rational expectations equilibrium. More precisely

$$
\begin{aligned}
\mu-p & =R P^{s t d d}(\widetilde{x})+\frac{1}{4} \frac{\left(\theta_{1}-\theta_{2}\right)^{2}\left(\sigma^{2}+\sigma \varpi \rho\right)}{\theta_{1} \theta_{2} \bar{\theta}} \\
\nu-q & =R P^{s t d d}(\widetilde{y})+\frac{1}{4} \frac{\left(\theta_{1}-\theta_{2}\right)^{2}\left(\varpi^{2}+\sigma \varpi \rho\right)}{\theta_{1} \theta_{2} \bar{\theta}}
\end{aligned}
$$

where $R P^{\text {stdd }}(\widetilde{x})$ and $R P^{\text {stdd }}(\widetilde{y})$ denote the standard risk-premium for $\widetilde{x}$ and $\widetilde{y}$ in an homogenous beliefs setting.

As far as the market portfolio $(\widetilde{x}+\widetilde{y})$ is concerned, the market risk-premium $R P^{M}$ and the beliefs $\xi_{i}^{M}$ on the average market return are given by

$$
\begin{aligned}
\xi_{i}^{M} & =\xi-\frac{\left(\theta_{i}-\theta_{j}\right) \sigma_{M}^{2}}{4 \theta_{j} \bar{\theta}} \\
R P^{M} & =R P^{s t d d}(\widetilde{x}+\widetilde{y})+\frac{1}{4} \frac{\left(\theta_{1}-\theta_{2}\right)^{2} \sigma_{M}^{2}}{\theta_{1} \theta_{2} \bar{\theta}}
\end{aligned}
$$

where $\xi=\mu+\nu$ and $\sigma_{M}^{2}=\varpi^{2}+2 \rho \sigma \varpi+\sigma^{2}$ correspond respectively to the objective market portfolio return and variance. These formulas are exactly the same as in the one asset framework which means that the more risk tolerant (risk averse) agent is pessimistic (optimistic) at the aggregate level and the consensus belief is pessimistic at the aggregate level. The formulas for individual assets that are provided in the proposition are similar to those obtained in the one asset framework. However, for each asset, the variance term in the one-asset formula is replaced by the covariance of the considered asset payoffs with the market portfolio payoffs. Recall that in the Walrasian setting (CAPM setting), the equilibrium price for a given asset depends on the covariance of the payoffs of this asset with the payoffs of the market portfolio and not on the total variance of the asset payoffs. Since the agents modify their beliefs in order to manipulate the prices, it is natural to obtain optimal beliefs that depend on the covariance with the market portfolio and not on the total variance. The aggregate level properties (pessimism, correlation between pessimism and risk tolerance,...) are then retrieved at the individual assets level as far as these assets are positively correlated with the market portfolio.

It is interesting to note that these effects are more pronounced for the riskier asset. 
Intuitively, the strategic behaviour leads to more beliefs dispersion for the riskier asset and hence to a more pronounced impact on the market for the riskier asset.

\section{Conclusion}

The introduction of strategic interaction provides a rationale for belief heterogeneity; it leads to beliefs that are subjective, heterogeneous and antagonistic. The selection of strategic beliefs is governed by very precise rules. These beliefs must be related to the individual level of risk-aversion: the beliefs of more risk-averse agents exhibit optimism and/or overconfidence and the beliefs of more risk-tolerant agents exhibit pessimism and/or doubt. As a consequence, there is a positive correlation between pessimism/doubt and risk-tolerance. In a setting with exponential utility and normal distributions, the average belief exhibits pessimism and/or doubt as well as the consensus belief. This is compatible with the observation that subjects in experimental and empirical studies exhibit a dose of pessimism (Wakker, 2001, Ben Mansour et al., 2006, Giordani and Söderlind, 2006). This induced pessimism/doubt of investors leads to higher risk premia, which is interesting in light of the equity premium puzzle. It is also helpful to explain the purchase of vastly overpriced insurance contracts or the large short run returns of IPOs.

This work suggests further investigation in several directions. First, in this paper we have let aside information asymmetry and heterogeneity in order to focus on the impact of strategic interactions on individual beliefs and from there on equilibrium prices and allocations. It would be useful to consider a more general model including both a strategic use of private information and a strategic choice of beliefs. Second, we have only considered totally ordered families of possible subjective distributions for the risky asset payoffs. In particular, all beliefs deformations can be interpreted in terms of pessimism/optimism or in terms of doubt/overconfidence. It would be interesting to consider more general possible deformations of the objective distribution in particular in terms of higher order moments (as skewness and kurtosis). Finally, it would be interesting to analyze a model with a large number of players. Since beliefs are built strategically, we may argue that the impact of the strategic behaviour will vanish when the number of players becomes large. However, "the real issue is not so much how many (agents) there are, but to what extent (agents) cluster in their beliefs" [Shefrin, 2005, p216]. Situations with a small number of clusters would correspond to situations with a small number of leaders (highly concentrated decision power, presence of gurus...). This should then lead to extend the model in order to take into account leadership, beliefs contagion and herding behaviour.

\section{Appendix}




\section{Proof of Proposition 3}

2. Let us first analyze the game $\Gamma$. The expected utility of agent $i$ at the Walras equilibrium, given the belief $\mu_{j}$ of agent $j, j \neq i$, can be written

$$
\begin{aligned}
U_{i}\left(\mu_{1}, \mu_{2}\right) & =E\left[-\exp \left(-\frac{\frac{1}{2} p\left(\mu_{1}, \mu_{2}\right)+\alpha_{i}\left(\mu_{1}, \mu_{2}\right)\left(\widetilde{x}-p\left(\mu_{1}, \mu_{2}\right)\right)}{\theta_{i}}\right)\right] \\
& =-\exp \left[-\left(\frac{\frac{1}{2} p\left(\mu_{1}, \mu_{2}\right)+\alpha_{i}\left(\mu_{1}, \mu_{2}\right)\left(\mu-p\left(\mu_{1}, \mu_{2}\right)\right)}{\theta_{i}}+\frac{1}{2}\left(\frac{\alpha_{i}\left(\mu_{1}, \mu_{2}\right)}{\theta_{i}}\right)^{2} \sigma^{2}\right)\right]
\end{aligned}
$$

where $\alpha_{i}\left(\mu_{1}, \mu_{2}\right)=\theta_{i} \frac{\mu_{i}-p\left(\mu_{1}, \mu_{2}\right)}{\sigma^{2}}$. We now have an explicit expression for the payoff functions of both agents. The problem of agent $i$ is then to maximize this payoff with respect to $\mu_{i}$, the strategy $\mu_{j}$ of the other player being given.

Maximizing $U_{i}\left(\mu_{1}, \mu_{2}\right)$ with respect to $\mu_{i}$ amounts to maximizing

$$
A_{i}\left(\mu_{1}, \mu_{2}\right)=\frac{1}{2} p\left(\mu_{1}, \mu_{2}\right)+\alpha_{1}\left(\mu_{1}, \mu_{2}\right)\left(\mu-p\left(\mu_{1}, \mu_{2}\right)\right)-\frac{1}{2} \frac{1}{\theta_{1}}\left(\alpha_{1}\left(\mu_{1}, \mu_{2}\right)\right)^{2} \sigma^{2} .
$$

This program is concave and the maximum is reached for $\mu_{i}$ such that $\frac{d A_{i}}{d \mu_{i}}\left(\mu_{i}\right)=0$. This corresponds to the best response $B R_{i}\left(\mu_{j}\right)$ of agent $i$ when agent $j$ plays $\mu_{j}$ and we have

$$
B R_{i}\left(\mu_{j}\right)=\frac{2 \mu \theta_{j}\left(\theta_{1}+\theta_{2}\right)+2 \theta_{i} \theta_{j} \mu_{j}+\sigma^{2}\left(\theta_{j}-\theta_{i}\right)}{4 \theta_{j} \theta_{i}+2 \theta_{i}^{2}} .
$$

We then solve for $\left(\widehat{\mu}_{1}, \widehat{\mu}_{2}\right)$ such that $B R_{1}\left(\widehat{\mu}_{2}\right)=\widehat{\mu}_{1}$ and $B R_{2}\left(\widehat{\mu}_{1}\right)=\widehat{\mu}_{2}$. We obtain equations (9).

1. Let us now prove that this unique Nash equilibrium characterizes the limit of our evolutionary process. First, let us show that the set of strategies surviving iterated strict dominance in the game $\Gamma$ is reduced to the unique Nash equilibrium exhibited above. It is equivalent to prove this result for the game $\Gamma$ or for the game $\widetilde{\Gamma}$ defined by the same strategic sets as $\Gamma$ but with payoff functionals $A_{1}$ and $A_{2}$ instead of $U_{1}$ and $U_{2}$. It suffices then to show that $\widetilde{\Gamma}$ is a supermodular game (see e.g. Milgrom and Roberts, 1990, or Fudenberg and Tirole, 1991). Since our strategic sets are compact and our payoff functions are continuous, it suffices to check that $A_{i}\left(\mu_{1}, \mu_{2}\right)$ has increasing differences in $\left(\mu_{1}, \mu_{2}\right)$, for $i=1,2$ or equivalently that $\frac{\partial^{2} A_{i}}{\partial \mu_{1} \partial \mu_{2}}\left(\mu_{1}, \mu_{2}\right) \geq 0$. This is immediate.

Let us show now that any strictly dominated strategy is eliminated by the evolution process.

Let us consider $\mu_{1}$ that is stricly dominated by $\mu_{1}^{\prime}$ from agent 1 point of view. This means that $U_{1}\left(\mu_{1}, \mu_{2}\right)<U_{1}\left(\mu_{1}^{\prime}, \mu_{2}\right)$ for all $\mu_{2}$. We have then for every distribution $\Theta$ over $B, \int U_{1}\left(\mu_{1}, \mu_{2}\right) d \Theta<\int U_{1}\left(\mu_{1}^{\prime}, \mu_{2}\right) d \Theta$ and then $V_{1}\left(\mu_{1}, \Theta\right)<V_{1}\left(\mu_{1}^{\prime}, \Theta\right)$. Since $B$ is compact, $\Delta(B)$ is compact with respect to the weak-topology. We have then $V_{1}\left(\mu_{1}, \Theta\right)+k<$ $V_{1}\left(\mu_{1}^{\prime}, \Theta\right)$ for some $k>0$. Let us now consider some open subset $A$ (resp. $A^{\prime}$ ) of $B$ 
containing $\mu_{1}$ (resp. $\left.\mu_{1}^{\prime}\right)$ such that $V_{1}(\mu, \Theta)+\frac{k}{2}<V_{1}\left(\mu^{\prime}, \Theta\right)$ for all $\left(\mu, \mu^{\prime}\right) \in B \times B$ and all $\Theta \in \Delta(B)$. We have

$$
\frac{d}{d t} \ln \left(\frac{\Upsilon_{t}(A)}{\Upsilon_{t}\left(A^{\prime}\right)}\right)=\frac{\int_{A} V^{1}\left(\gamma, \Theta_{t}\right) d \Upsilon_{t}}{\Upsilon_{t}(A)}-\frac{\int_{A^{\prime}} V^{1}\left(\gamma, \Theta_{t}\right) d \Upsilon_{t}}{\Upsilon_{t}\left(A^{\prime}\right)}<-\frac{k}{2}
$$

which gives that $\frac{\Upsilon_{t}(A)}{\Upsilon_{t}\left(A^{\prime}\right)} \rightarrow 0$ and then $\Upsilon_{t}(A) \rightarrow 0$. For each strictly dominated strategy $\mu_{1}$, there exists then an open set $A$ such that $\mu_{1} \in A \subset B$ and such that $\Upsilon_{t}(A) \rightarrow 0$. Adapting the proof of Theorem 1 in Heifetz et al. (2007b) to non-symmetric payoffs, we obtain that the asymptotic distribution concentrates all the mass on the unique strategy that is not eliminated by iterated strict dominance, that is to say $\widehat{\mu}_{1}$.

3. and 4. Straightforward using Equations (9).

\section{Proof of Proposition 6}

The utility level of agent $i$ is given by $\left.E_{i}\left[u_{i}\left(\frac{1}{2} p+\alpha_{i}\left(p, \mu_{i}\right)(\widetilde{x}-p)\right)\right)\right]$ with $\alpha_{i}\left(p, \mu_{i}\right)=$ $\theta_{i} \frac{\mu_{i}-p}{\sigma^{2}}$. Then, for a given $p$, the agent maximizes $\theta_{i} \frac{\left(\mu_{i}-p\right)^{2}}{\sigma^{2}}$.

When $p>\frac{\mu+\bar{\mu}}{2}$, all the agents have the same belief $\underline{\mu}$ and the equilibrium price, if it exists, must satisfy $\bar{p}=\underline{\mu}-\frac{\sigma^{2}}{\theta_{1}+\theta_{2}}$ which is not compatible with the condition $p>\frac{\mu+\bar{\mu}}{2}$. When $p<\frac{\underline{\mu}+\bar{\mu}}{2}$, all the agents have the same belief $\bar{\mu}$ and the equilibrium price, if it exists, must satisfy $\bar{p}=\bar{\mu}-\frac{\sigma^{2}}{\theta_{1}+\theta_{2}}$ which is compatible with the condition $p<\frac{\mu+\bar{\mu}}{2}$ only if $\frac{\sigma^{2}}{\theta_{1}+\theta_{2}}>\frac{\bar{\mu}-\underline{\mu}}{2}$. When $p=\frac{\underline{\mu}+\bar{\mu}}{2}$, both agents may choose the same belief $\bar{\mu}$ leading to an equilibrium only if $\frac{\sigma^{2}}{\theta_{1}+\theta_{2}}=\frac{\bar{\mu}-\underline{\mu}}{2}$. They may also choose different beliefs. If agent 1 (resp. 2) chooses $\underline{\mu}$ (resp. $\bar{\mu})$, the market clearing condition leads to

$$
\frac{\theta_{1} \underline{\mu}+\theta_{2} \bar{\mu}}{\theta_{1}+\theta_{2}}-\frac{\sigma^{2}}{\theta_{1}+\theta_{2}}=\frac{\underline{\mu}+\bar{\mu}}{2}
$$

If $\mu=\frac{\underline{\mu}+\bar{\mu}}{2}$ and $\theta_{1}<\theta_{2}$, then $\frac{\theta_{1} \mu+\theta_{2} \bar{\mu}}{\theta_{1}+\theta_{2}}>\mu$.

\section{Proof of Proposition 7}

1. It is well known that due to Inada conditions, the demand function is characterized by the following first order condition

$$
E^{\mu}\left[(\tilde{x}-p) u_{i}^{\prime}\left(\alpha_{i}(p, \mu)(\tilde{x}-p)+\frac{1}{2} p\right)\right]=0
$$

and the partial derivatives of $\alpha_{i}(p, \mu)$ with respect to $p$ and $\mu$ are given by

$$
\begin{aligned}
\frac{\partial \alpha_{i}}{\partial p}(p, \mu) & =-\frac{E^{\mu}\left[\left(\frac{1}{2}-\alpha_{i}(p, \mu)\right)(\tilde{x}-p) u_{i}^{\prime \prime}(c(p, \mu))-u_{i}^{\prime}(c(p, \mu))\right]}{E^{\mu}\left[(\tilde{x}-p)^{2} u_{i}^{\prime}(c(p, \mu))\right]}, \\
\frac{\partial \alpha_{i}}{\partial \mu}(p, \mu) & =-\frac{\left.\frac{\partial}{\partial \mu} E^{\mu}\left[(\tilde{x}-p) u_{i}^{\prime}(c(p, \mu))\right]\right|_{\left(\mu, p, \alpha_{i}(p, \mu)\right)}}{E^{\mu}\left[(\tilde{x}-p)^{2} u_{i}^{\prime}(c(p, \mu))\right]},
\end{aligned}
$$


with $c(p, \mu)=\alpha_{i}(p, \mu)(\tilde{x}-p)+\frac{1}{2} p$. Letting $\widetilde{c}$ denote $c(p, \mu)$, remark that

$$
E^{\mu}\left[\left(\frac{1}{2}-\alpha_{i}(p, \mu)\right)(\tilde{x}-p) u_{i}^{\prime \prime}(\widetilde{c})-u_{i}^{\prime}(\widetilde{c})\right]=E^{\mu}\left[-u_{i}^{\prime}(\widetilde{c})-\widetilde{c} u_{i}^{\prime \prime}(\widetilde{c})+\frac{1}{2} \widetilde{x} u_{i}^{\prime \prime}(\widetilde{c})\right] .
$$

Hence, $\frac{\partial \alpha_{i}}{\partial p}(p, \mu)$ is negative. Furthermore, $(\tilde{x}-p) u_{i}^{\prime}(\widetilde{c})=\frac{1}{\alpha_{i}(p, \mu)} \widetilde{c} u^{\prime}(\widetilde{c})-\frac{1}{2} \frac{p}{\alpha_{i}(p, \mu)} u^{\prime}(\widetilde{c})$ and is then increasing. By the first-stochastic dominance property, we have $\frac{\partial \alpha_{i}}{\partial \mu}(p, \mu) \geq 0$.

We have then

$$
\frac{\partial p}{\partial \mu_{i}}=-\frac{\frac{\partial \alpha_{i}}{\partial \mu_{i}}\left(p, \mu_{i}\right)}{\frac{\partial \alpha_{1}}{\partial p}\left(p, \mu_{1}\right)+\frac{\partial \alpha_{2}}{\partial p}\left(p, \mu_{2}\right)}
$$

hence $\frac{\partial p}{\partial \mu_{i}} \geq 0, i=1,2$.

For $i \neq j$, we have $\frac{\partial \alpha_{i}^{*}\left(\mu_{1}, \mu_{2}\right)}{\partial \mu_{i}}=\frac{\partial \alpha_{i}\left(p, \mu_{i}\right)}{\partial \mu_{i}}+\frac{\partial \alpha_{i}\left(p, \mu_{i}\right)}{\partial p} \frac{\partial p}{\partial \mu_{i}}=\frac{\frac{\partial \alpha_{i}}{\partial \mu_{i}}\left(p, \mu_{i}\right) \frac{\partial \alpha_{j}}{\partial p}\left(p, \mu_{j}\right)}{\frac{\partial \alpha_{1}}{\partial p}\left(p, \mu_{1}\right)+\frac{\partial \alpha_{2}}{\partial p}\left(p, \mu_{2}\right)} \geq 0$.

2. If the game $\Gamma$ has an interior Nash equilibrium $\left(\hat{\mu}_{1}, \hat{\mu}_{2}\right)$, the first-order condition for agent $i$ at $\left(\hat{\mu}_{1}, \hat{\mu}_{2}\right)$ gives

$$
E\left[\left(\frac{\partial \alpha_{i}^{*}}{\partial \mu_{i}}(\tilde{x}-p)+\left(\frac{1}{2}-\alpha_{i}^{*}\right) \frac{\partial p}{\partial \mu_{i}}\right) u_{i}^{\prime}\left(\alpha_{i}^{*}(\tilde{x}-p)+\frac{1}{2} p\right)\right]=0 .
$$

If $\frac{1}{2}-\alpha_{i}^{*}\left(\hat{\mu}_{1}, \hat{\mu}_{2}\right) \leq 0$ then $\left(\frac{1}{2}-\alpha_{i}^{*}\left(\hat{\mu}_{1}, \hat{\mu}_{2}\right)\right) \frac{\partial p}{\partial \mu_{i}} \leq 0$, hence $E\left[\left(\tilde{x}-p\left(\hat{\mu}_{1}, \hat{\mu}_{2}\right)\right) u_{i}^{\prime}\right] \geq 0$. As previously, by the first-order stochastic dominance property we obtain $\hat{\mu}_{i} \leq 0$. Analogously $\frac{1}{2}-\alpha_{i}^{*}\left(\hat{\mu}_{1}, \hat{\mu}_{2}\right) \geq 0$ leads to $\hat{\mu}_{i} \geq 0$. We have then proved that the agent for which $\alpha_{i}^{*}\left(\hat{\mu}_{1}, \hat{\mu}_{2}\right) \geq \frac{1}{2}$ (resp. $\left.\alpha_{i}^{*}\left(\hat{\mu}_{1}, \hat{\mu}_{2}\right) \leq \frac{1}{2}\right)$ is pessimistic (resp. optimistic).

3 . If one of the utility functions (let us say $u_{1}$ ) is more risk-averse than the other one in the sense of Arrow-Pratt, let us prove that $\alpha_{1}^{*}\left(\hat{\mu}_{1}, \hat{\mu}_{2}\right) \leq \frac{1}{2}$. If this is not the case, we have $\alpha_{2}^{*}\left(\hat{\mu}_{1}, \hat{\mu}_{2}\right) \leq \frac{1}{2}$ and agent 2 is optimistic while agent 1 is pessimistic. We have then

$$
\frac{1}{2}<\alpha_{1}\left(p\left(\hat{\mu}_{1}, \hat{\mu}_{2}\right), \hat{\mu}_{1}\right) \leq \alpha_{2}\left(p\left(\hat{\mu}_{1}, \hat{\mu}_{2}\right), \hat{\mu}_{1}\right)
$$

because agent 1 is more risk-averse. Furthermore we have $\alpha_{2}\left(p\left(\hat{\mu}_{1}, \hat{\mu}_{2}\right), \hat{\mu}_{1}\right) \leq \alpha_{2}\left(p\left(\hat{\mu}_{1}, \hat{\mu}_{2}\right), \hat{\mu}_{2}\right)$ because $\hat{\mu}_{2}$ is larger than $\hat{\mu}_{1}$. We would have then $\alpha_{2}^{*}\left(\hat{\mu}_{1}, \hat{\mu}_{2}\right)>\frac{1}{2}$ which contradicts our assumption.

4. See Proof of Proposition 3 (point 1).

\section{Proof of Proposition 8}

2. Let us first characterize the Nash equilibrium in the game $\Gamma$. Agent $i$ maximizes

$$
A_{i}\left(\sigma_{1}, \sigma_{2}\right)=\frac{1}{2} p+\alpha_{i}(\mu-p)-\frac{1}{2} \frac{1}{\theta_{i}}\left(\alpha_{i}\right)^{2} \sigma^{2}
$$

with respect to $\sigma_{i}$ where $p$ and $\alpha_{i}$ both depend on $\sigma_{i}$ and are given by $p=\mu-\left(\frac{\theta_{1}}{\sigma_{1}^{2}}+\frac{\theta_{2}}{\sigma_{2}^{2}}\right)^{-1}$ and $\alpha_{i}=\theta_{i} \frac{\mu-p}{\sigma_{i}^{2}}$. 
This problem is concave in $\left(\frac{\theta_{1}}{\sigma_{1}^{2}}+\frac{\theta_{2}}{\sigma_{2}^{2}}\right)^{-1}$. Setting $\frac{d A_{i}}{d \sigma_{i}}\left(\widehat{\sigma}_{1}, \widehat{\sigma}_{2}\right)=0$ leads to

$$
\widehat{\sigma}_{i}^{2}=\frac{2}{3} \sigma^{2}+\frac{1}{3}\left(\frac{\theta_{i}}{\theta_{j}} \widehat{\sigma}_{j}^{2}\right)
$$

The resulting beliefs are then given by Equations (14). Equation (15) follows.

Since

$$
\left|\left(\frac{\theta_{1}-\theta_{2}}{4 \theta_{2}}\right)\left(\frac{4 \theta_{1}}{\theta_{2}-\theta_{1}}\right)\right|=\frac{\theta_{1}}{\theta_{2}}
$$

the more risk-tolerant agent exhibits more doubt than the less risk-tolerant agent exhibits overfidence.

1. In the "disagreement on the variance" case, the game is not supermodular. However, as in Section 3, the set of strategies surviving iterated strict dominance is reduced to the unique Nash equilibrium exhibited above. Indeed, let us denote by $\left[\underline{\sigma}_{1}^{2 n}, \bar{\sigma}_{1}^{2 n}\right]$ the set of strategies surviving after $n$ rounds of strict dominance (these sets are constructed by induction and the construction will be clearer at the end of the argument) and let us denote by

$$
\begin{aligned}
& \underline{\sigma}_{2}^{2 n+1}=\arg \max A_{2}\left(\underline{\sigma}_{1}^{2 n}, \sigma_{2}\right)=\sqrt{\frac{2}{3} \sigma^{2}+\frac{1}{3}\left(\frac{\theta_{2}}{\theta_{1}}\left(\underline{\sigma}_{1}^{2 n}\right)^{2}\right)} \\
& \bar{\sigma}_{2}^{2 n+1}=\arg \max A_{2}\left(\bar{\sigma}_{1}^{2 n}, \sigma_{2}\right)=\sqrt{\frac{2}{3} \sigma^{2}+\frac{1}{3}\left(\frac{\theta_{2}}{\theta_{1}}\left(\bar{\sigma}_{1}^{2 n}\right)^{2}\right)} .
\end{aligned}
$$

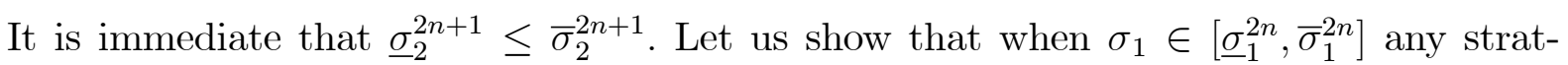
egy for agent 2 that lies outside $\left[\underline{\sigma}_{2}^{2 n+1}, \bar{\sigma}_{2}^{2 n+1}\right]$ is stricly dominated by a strategy inside this interval. For a given $\sigma_{1} \in\left[\underline{\sigma}_{1}^{2 n}, \bar{\sigma}_{1}^{2 n}\right]$, the function $A_{2}\left(\sigma_{1}, \sigma_{2}\right)$ is increasing until $\sqrt{\frac{2}{3} \sigma^{2}+\frac{1}{3}\left(\frac{\theta_{2}}{\theta_{1}}\left(\sigma_{1}\right)^{2}\right)}$ and then decreasing. Since $\sqrt{\frac{2}{3} \sigma^{2}+\frac{1}{3}\left(\frac{\theta_{2}}{\theta_{1}}\left(\sigma_{1}\right)^{2}\right)} \in\left[\underline{\sigma}_{2}^{2 n+1}, \bar{\sigma}_{2}^{2 n+1}\right]$ this means that, for any $\sigma_{2}<\underline{\sigma}_{2}^{2 n+1}$, we have $A_{2}\left(\sigma_{1}, \sigma_{2}\right)<A_{2}\left(\sigma_{1}, \underline{\sigma}_{2}^{2 n+1}\right)$ and $\sigma_{2}$ is then strictly dominated by $\underline{\sigma}_{2}^{2 n+1}$. Similarly, any $\sigma_{2}>\bar{\sigma}_{2}^{2 n+1}$ is dominated by $\bar{\sigma}_{2}^{2 n+1}$. The set $\left[\underline{\sigma}_{1}^{2 n+2}, \bar{\sigma}_{1}^{2 n+2}\right]$ is constructed similarly switching the roles between agent 1 and 2 . We have then decreasing sets of surviving strategies and it is easy to show that, at the limit, we must have $\underline{\sigma}_{2}^{\infty}=\sqrt{\frac{2}{3} \sigma^{2}+\frac{1}{3}\left(\frac{\theta_{2}}{\theta_{1}}\left(\underline{\sigma}_{1}^{\infty}\right)^{2}\right)}$ and $\underline{\sigma}_{1}^{\infty}=\sqrt{\frac{2}{3} \sigma^{2}+\frac{1}{3}\left(\frac{\theta_{1}}{\theta_{2}}\left(\underline{\sigma}_{2}^{\infty}\right)^{2}\right)}$. We know from 2 . that these equations in $\left(\underline{\sigma}_{1}^{\infty}, \underline{\sigma}_{2}^{\infty}\right)$ admit a unique solution $\left(\widehat{\sigma}_{1}, \widehat{\sigma}_{2}\right)$ and we have then $\underline{\sigma}_{1}^{\infty}=\widehat{\sigma}_{1}$ and $\underline{\sigma}_{2}^{\infty}=\widehat{\sigma}_{2}$. The same argument gives us $\bar{\sigma}_{1}^{\infty}=\widehat{\sigma}_{1}$ and $\bar{\sigma}_{2}^{\infty}=\widehat{\sigma}_{2}$ and the set of surviving strategy is reduced to $\left(\widehat{\sigma}_{1}, \widehat{\sigma}_{2}\right)$.

3. and 4. Straightforward using equations (14).

\section{Proof of Proposition 9}


2. In the setting of the proposition, agent $i$ maximizes

$A_{i}\left(\mu_{1}, \nu_{1}, \mu_{2}, \nu_{2}\right)=\frac{1}{2} p+\alpha_{i}(\mu-p)+\frac{1}{2} q+\beta_{i}(\nu-q)-\frac{1}{2} \frac{1}{\theta_{i}}\left(\alpha_{i}^{2} \sigma^{2}+\beta_{i}^{2} \varpi^{2}+2 \alpha_{i} \beta_{i} \sigma \varpi \rho\right)$

with respect to $\left(\mu_{i}, \nu_{i}\right)$ taking $\left(\mu_{j}, \nu_{j}\right)=\left(\widehat{\mu}_{j}, \widehat{\nu}_{j}\right), j \neq i$, as given. The maximization programs under consideration are concave. Setting $\frac{d A_{i}}{d \mu_{i}}=\frac{d A_{i}}{d \nu_{i}}=0$, for $i=1,2$, leads to

$$
\widehat{\mu}_{i}=\mu-\frac{\left(\theta_{i}-\theta_{j}\right)\left(\sigma^{2}+\sigma \varpi \rho\right)}{4 \theta_{j} \bar{\theta}}, \quad \widehat{\nu}_{i}=\nu-\frac{\left(\theta_{i}-\theta_{j}\right)\left(\varpi^{2}+\sigma \varpi \rho\right)}{4 \theta_{j} \bar{\theta}}
$$

which is the unique Nash equilibrium. We then conclude as in the proof of Proposition 3 .

3. Straightforward using 2 .

1. Let us show that the game $\Gamma$ where agent 1 chooses her strategy $b^{1}=\left(\mu_{1}, \nu_{1}\right)$ in her strategic set $B=[\underline{\mu}, \bar{\mu}] \times[\underline{\nu}, \bar{\nu}]$ and agent 2 chooses her strategy $b^{2}=\left(\mu_{2}, \nu_{2}\right)$ in the same strategic set, is a supermodulat game when $B$ is endowed with the natural partial order $\preceq$ defined for $b=(\mu, \nu)$ and $b^{\prime}=\left(\mu^{\prime}, \nu^{\prime}\right)$ by $b \preceq b^{\prime}$ if $\mu \leq \mu^{\prime}$ and $\nu \leq \nu^{\prime}$. For this purpose, it suffices to check that $A_{i}\left(b^{1}, b^{2}\right)$ has increasing differences, i.e. for all $b_{1}^{\prime} \geq b_{1}$ and $b_{2}^{\prime} \geq b_{2}$ we have $A_{i}\left(b_{1}^{\prime}, b_{2}^{\prime}\right)-A_{i}\left(b_{1}, b_{2}^{\prime}\right) \geq A_{i}\left(b_{1}^{\prime}, b_{2}\right)-A_{i}\left(b_{1}, b_{2}\right)$. Easy computations give us $\frac{\partial^{2} A_{i}}{\partial \mu_{i} \partial \mu_{j}} \geq 0, \frac{\partial^{2} A_{i}}{\partial \nu_{i} \partial \mu_{j}} \geq 0, \frac{\partial^{2} A_{i}}{\partial \mu_{i} \partial \nu_{j}}$ and $\frac{\partial^{2} A_{i}}{\partial \nu_{i} \partial \nu_{j}} \geq 0$ which is sufficient to prove that $A_{i}$ has increasing differences. The rest of the proof is similar to the proof of Proposition 3. 


\section{References}

[1] Abel, A., 1989. Asset prices under heterogeneous beliefs: implications for the equity premium. Mimeo, University of Pennsylvania.

[2] Abel, A., 2002. An exploration of the effects of pessimism and doubt on asset returns. Journal of Economic Dynamics and Control, 26, 1075-1092.

[3] Akerlof, G. and W.T. Dickens, 1982. The economic consequences of cognitive dissonance. American Economic Review, 72, 3, 307-319.

[4] Alchian, R, 1950. Uncertainty, evolution and economic theory. Journal of Political Economy, 58, 211-221.

[5] Aumann, R.J., 1976. Agreeing to Disagree. Annals of Statistics, 4, 1236-1239.

[6] Baumeister, R. F., 1998. The Self. In D. Gilbert, S. Fiske, and G. Lindzey, eds., The Handbook of Social Psychology. New York: McGraw-Hill, 680-740.

[7] Ben Mansour, S., Jouini, E. and C. Napp, 2006. Is There a "Pessimistic" Bias in Individual Beliefs? Evidence from a Simple Survey. Theory and Decision, 61, 345362 .

[8] Ben Mansour, S., Jouini, E., Marin, J.-M., Napp C. and C. Robert, 2008. Are risk tolerant agents more pessimistic ? A Bayesian estimation approach. Journal of Applied Econometrics, 843-860.

[9] Benabou, R. and J. Tirole, 2002. Self-confidence and personal motivation. Quarterly Journal of Economics, 117, 871-915.

[10] Brunnermeier, M.K., and J. Parker, 2005. Optimal Expectations. American Economic Review, 95, 4, 1092-1118.

[11] Brunnermeier, M.K., Gollier, C. and J. Parker, 2007. Optimal Beliefs, Asset Prices, and the Preference for Skewed Returns. American Economic Review, 97, 159-165.

[12] Calvet, L., Grandmont, J.-M. and I. Lemaire, 2002. Aggregation of heterogeneous beliefs and asset pricing in complete financial markets. Working Paper.

[13] Cressman R., Hofbauer, J. and F. Riedel, 2006. Stability of the replicator equation for a single species with a multi-dimensional continuous trait space. Journal of Theoretical Biology, 239, 237-288.

[14] Cutler, D.M. and R. Zeckhauser, 2004. Extending the Theory to Meet the Practice of Insurance. In Robert E. Litan and Richard Herring (eds.), Brookings-Wharton Papers on Financial Services, 1-53. 
[15] Darrough, M.N. and T. Russell, 2002. A positive model of earnings forecasts: Top down versus bottom up. Journal of Business 75 (1), 127-152.

[16] Detemple, J. and S. Murthy, 1994. Intertemporal asset pricing with heterogeneous beliefs. Journal of Economic Theory, 62, 294-320.

[17] Dreman, D.N. and M. Berry, 1995. Analyst forecasting errors and their implications for security analysts. Financial Analysts Journal 51, 30-40.

[18] Duchin, R. and M. Levy, 2010. Disagreement, Portfolio Optimization and Excess Volatility. Journal of Financial and Quantitative Analysis, 45, 623-640

[19] Duval, T. and P. Silvia, 2002. Self-Awareness, Probability of Improvement, and the Self-serving Bias. Journal of Personality and Social Psychology, 82, 49-61.

[20] Fama, E. and K. French, 2007. Disagreement, Tastes and Asset Prices. Journal of Financial Economics, 83, 667-689.

[21] Fiske, S. and S. Taylor, 1991. Social Cognition. New York: McGraw-Hill.

[22] Francis, J. and D. Philbrick, 1993. Analysts' decisions as products of a multi-task environment. Journal of Accounting Research 31, 216-230.

[23] Fried, D. and D. Givoly, 1982. Financial Analysts' Forecasts of Earnings: A Better Surrogate for Earnings Expectations. Journal of Accounting and Economics 4, 85107.

[24] Friedman, M., 1953. Essays in positive economics. University of Chicago Press.

[25] Fudenberg, D. and J. Tirole, 1991. Game Theory. MIT Press.

[26] Giordani, P. and P. Söderlind, 2006. Is there evidence of pessimism and doubt in subjective distributions? Implications for the risk premium puzzle. Journal of Economic Dynamics and Control, 30, 1027-1043.

[27] Gollier, C., 2001. The Economics of Risk and Time, MIT Press.

[28] Gollier, C., 2005. Optimal illusions and decision under risk. IDEI Working Paper, 340.

[29] Gollier, C., 2007. Whom Should We Believe? Aggregation and Heterogeneous Beliefs. Journal of Risk and Uncertainty, 35, 107-127.

[30] Gollier, C. and A. Muermann, 2010. Optimal choice and beliefs with ex Ante savoring and Ex Post disappointment. Management Science, 56, 1272-1284. 
[31] Heifetz, A., Shannon, C. and Y. Spiegel, 2007a. What to maximize if you must. Journal of Economic Theory, 133, 31-57.

[32] Heifetz, A., Shannon, C. and Y. Spiegel, 2007b. The Dynamic Evolution of Preferences. Economic Theory, 32, 251-286.

[33] Hirshleifer, D., 2001. Investor psychology and asset pricing. Journal of Finance 56, 1533-1597.

[34] Hvide, H. K., 2002. Pragmatic beliefs and overconfidence. Journal of Economic Behaviour and Organization, 48, 15-28.

[35] Ibbotson, R.G., and J.R. Ritter, 1995. Initial Public Offerings. Chapter 30 of NorthHolland Handbooks in Operations Research and Management Science, Vol. 9: Finance. Edited by R. Jarrow, V. Maksimovic, and W. Ziemba.

[36] Jouini, E. and C. Napp, 2006. Heterogeneous beliefs and asset pricing in discrete time: An analysis of pessimism and doubt. Journal of Economic Dynamics and Control 30, 1233-1260.

[37] Jouini, E. and C. Napp, 2007. Consensus consumer and intertemporal asset pricing under heterogeneous beliefs. Review of Economic Studies, 74, 1149-1174.

[38] Kang, S., O’Brien, J. and K. Sivaramakrishnan, 1994. Analysts' interim earnings forecasts: Evidence on the forecasting process. Journal of Accounting Research 32, 103-112.

[39] Kyle, A., 1989. Informed speculation with imperfect competition. The Review of Economic Studies, Vol. 56, 3, 317-355.

[40] Landsberger, M., and I. Meilijson, 1990. Demand for risky financial assets: A portfolio analysis. Journal of Economic Theory, 50, 204-213.

[41] Lintner, J., 1965. The valuation of risky assets and the selection of risky investments in stock portfolios and capital budgets. Review of Economics and Statistics, 47: 13-37.

[42] McNichols, M. and P.C. O'Brien, 1997. Self-Selection and Analyst Coverage. Journal of Accounting Research 35, 167-199.

[43] Markowitz, H., 1952. Portfolio selection. Journal of Finance, 7, 77-91.

[44] Mehra, R. and E. Prescott, 1985. The Equity Premium: A Puzzle. Journal of Monetary Economics, 15, 145-162. 
[45] Milgrom, P. and J. Roberts, 1990. Rationalizability, learning, and equilibrium in games with strategic complementarities. Econometrica, 58, 1255-77.

[46] O'Brien, P.C., 1988. Analysts Forecasts as Earnings Expectations. Journal of Accounting and Economics 10, 53-88.

[47] Oechssler, J. and F. Riedel, 2001. Evolutionary dynamics on infinite strategy spaces. Economic Theory, 17, 141-162.

[48] Rabin, M., 1998. Psychology and Economics. Journal of Economic Literature 36, $11-46$.

[49] Russel, B., 1945. A History of Western Philosophy. Simon and Schuster, New York.

[50] Sandroni, A., 2000. Do markets favor agents able to make accurate prediction. Econometrica, 68, 1303-1341.

[51] Savage, L.J., 1954. The Foundation of Statistics. Wiley, New York.

[52] Schelling, T., 1960. The strategy of conflict. Harvard University Press, Cambridge, MA.

[53] Schipper, K., 1991. Commentary on analysts' forecasts. Accounting Horizons, 5, 105-121.

[54] Sharpe, W.F., 1964. Capital Asset Prices: A Theory of Market Equilibrium Under Conditions of Risk. Journal of Finance, 19, 425-442.

[55] Shefrin, H., 2005. A behavioural Approach to Asset Pricing, Academic Press, Advanced Finance Series.

[56] Shiller, R. J., 2000. Irrational exuberance. Princeton University Press.

[57] Van den Steen, E., 2004. Rational Overoptimism (and Other Biases). The American Economic Review, 94, 1141-1151.

[58] Wakker, P., 2001. Testing and Characterizing Properties of Nonadditive Measures through Violations of the Sure-Thing Principle. Econometrica 69, 1039-1059.

[59] Wenglert, L. and A.S. Rosen, 2000. Measuring optimism-pessimism from beliefs about future events. Personality and Individual Differences 28, 717-728.

[60] Williams, J.T., 1977. Capital Asset Prices with Heterogeneous Beliefs. Journal of Financial Economics, 5, 219-239.

[61] Yan, H., 2010. Is Noise Trading Cancelled out by Aggregation ? Management Science, 56, 1047-1059. 
[62] Zuckerman, M., 1979. Attribution of success and failure revisited, or: The motivational bias is alive and well in attribution theory. Journal of Personality, 47, 245-287. 